SB 945

. C8 S2

Copy 1

\title{
THE BOLL WEEVIL
}

\author{
AND
}

COTTON CROP

\section{OF TEXAS}

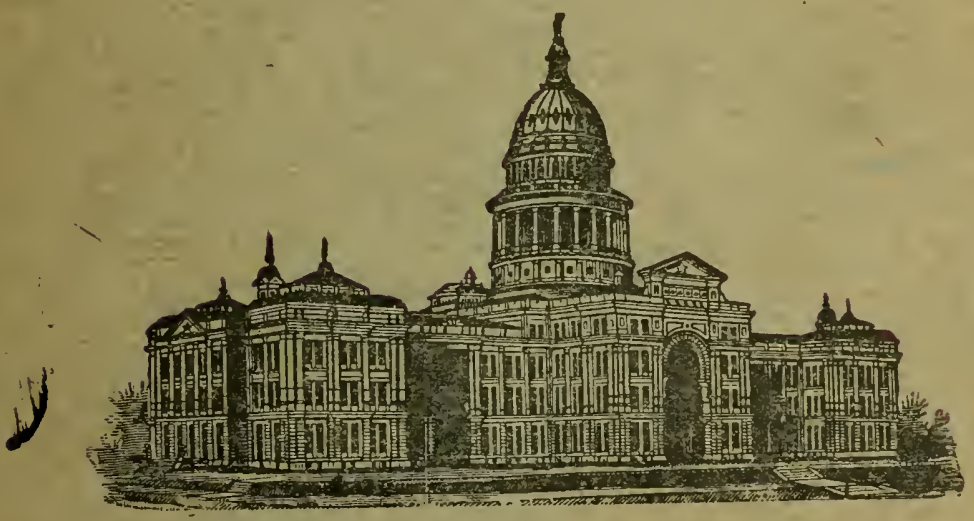

\section{W. J. CLAY \\ COMUISSIONER.}



DEPARTMENT OF

$168-815-1 / 2 \mathrm{~m}$.

A(iRIOUITURE, INSURANOE, STATISTICA ANI) HIS'TORY AUSTIN, TEXAS.

\title{
A STATISTICAL STUI)Y
}

OF TIF:

\author{
DECREASE IN THE TEXAS COT'TON CROP
}

DIF: TO

\section{THE VEXICAN COTTON BOLL WEEVIL \\ AND THF}

\section{CO'TON ACREAGE OF TEXAS}

1899 TO 1904 INCLUSIVE.

By E. DWIGHT SANDERSON

Recently State Entomolorist.

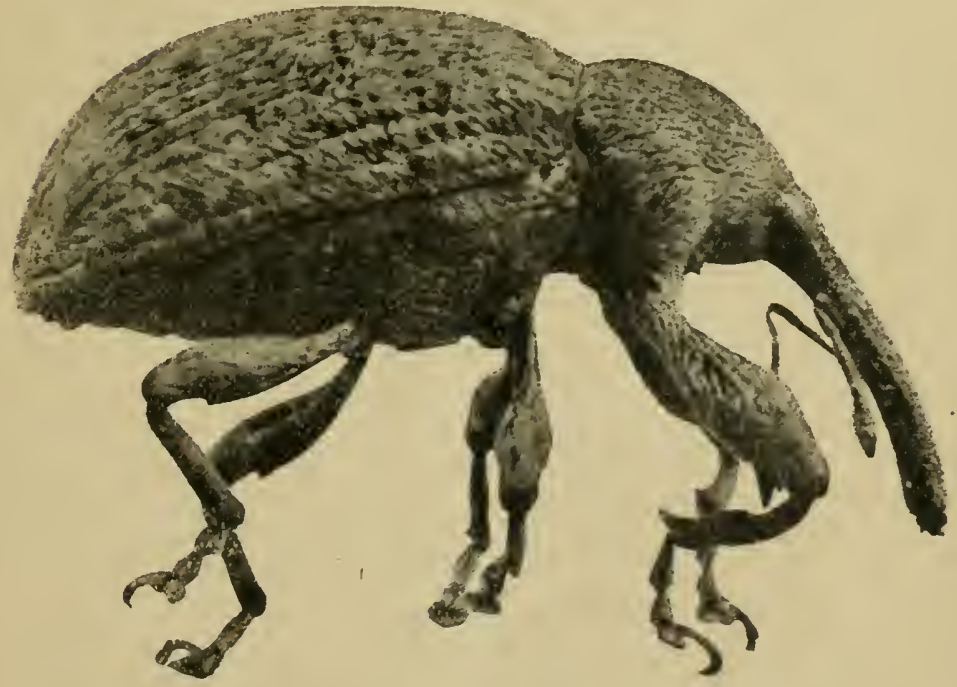

The Mexican Cotton Boll Weevil Magnified is Time's.

A USTIN, TEXAS:

STATE PRINTINA COMPANY

1605 . 


$$
53^{3^{4 x^{5}}} \cdot c^{520}
$$




\section{INTRODUCTORY.}

Toward mitigating the losses occasioned by the cotton boll weevil accurate statistics of the cotton crop for each county of the infested area are of the utmost importance, for the loss due to this insect is now'so great that it must exercise an increasingly important influence upon the market price of cotton. A short crop means a higher price, but it not infrequently happens that the higher price is not realized by the farmer, the rise in price taking place after he has sold the bulk of the crop to parties who realize the profit. If the farmer received the price for his cotton which the market supply warranted the loss occasioned by the weevil would in many instances be largely palliated, for there is more profit in but $1 / 4$ bale per acre at 10 cents per pound, as in 0.4 bale at 6 cents. The quicker and more accurately that the actual amount of damage due to the weevil and the total product of the infested area can be forecasted each year and the statistics placed in the hands of the farmer, the more likely he is to sell his crop intelligently and to realize the most that the market will warrant. Such statistics will of course be of most value to the infested area in years of an average or short crop in other States and of less value in years of a bumper crop. These facts are of course almost axiomatic, but may well receive special consideration at the present time when the immense crop of 1904 has perhaps somewhat obscured the probable influence of the boll weevil as a factor in the amount of the cotton crop for 1905 and future years.

Past Estimates.-The first estimate of the amount of damage by the weevil was made by Mr. C. H. Tyler Townsend, then field agent of the Division of Entomology of the United States Department of Agriculture, in December, 1894. (Insect Life, Vol. VII, page 308.) He estimated that one-sixth of the cotton producing region of the State was infested, the damage in the infested region being 90 per cent and this amounting to 15 per cent of the crop of the State. This was undoubtedly quite excessive. The counties infested in 1894 and in 1899 formed but 5 per cent of the cotton acreage of the State and probably had about the same proportion in 1894. In 1899 they produced but 4 per cent of the total crop of the State.

In August, 1902, Prof. F. W. Mally, in his Report Upon the Boll Weevil, gave an estimate of the percentage of injury in each of the infested counties, making a total of 235,969 bales loss for 1902 . These estimates were made before any figures could be secured upon the total yield for that year and at a season when the injury by the weevil is at its worst. Consequently it is not surprising that the actual yield of the infested counties as shown in our tables indicates rather less injury than was anticipated by Prof. Mally for that year. Mr. W. D. Hunter, in charge of the boll weevil investigations of the United States 
Department of Agriculture, in January, 1903 (Farmers' Bulletin 163, U. S. Dept. Agr.), gave $\$ 10,000,000$ as a conservative estimate of the damage done in 1902, which is practically that given in Table 1.

In November, 1903, at the second Texas Cotton Convention at Dallas, Mr. Hunter estimated the injury for 1903 at about $\$ 15,000,000$. (Proceedings 2d Ann. Texas Cotton Conv. p. 74, and Farmers' Bulletin $189, \mathrm{p} .13$.

In December of the same year the writer made an estimate of the amount of damage done based upon a comparison of the crops of the infested and uninfested counties for 1902 and 1903 with that of 1899 as given in the bulletins of the United States Census. (Proceedings of the Society for Promotion of Agricultural Science, 1903, pp. 15\%-170, reprinted as Circular 8, Entomological Department, Texas Agr. Exp. Station, Apr., 1904.) Upon this basis a loss of 445,956 bales for 1902 and 500,000 for 1903 was indicated. At that time the writer became fully aware of the impossibility of making any accurate estimate of the damage done by the weevil without a knowledge of the actual acreage of each of the injured counties for each year since its infestation, with the average vield per acre prior to the advent of the weevil for each county. This is clearly shown by a comparison of the estimates in the years given below with those then made. Though the estimate for 1903 was approximately correct, that for 1902 was more than double the probable correct amount as now shown by the writer and previously by Hunter and Mally.

'I'o determine the injury done by the weevil with any degree of accuracy it is necessary to ascertain the yield per acre for each county in the injured and uninjured area and to compare this with the yield per acre of the same counties prior to injury by the weevil. This will give the decrease of production due to the weevil in the counties known to have been injured, except as influenced by storms, floods, and insects, such as the boll worm, which factors it is almost impossible to estimate in many cases.

Thanks to the excellent system of ginners' reports now employed by the United States Census Bureau it is possible to secure the actual product of each county for each year since 1899, with the greatest accuracy possible.

From $188 \%$ to 1895 the Commissioner of Agriculture of Texas in his annual reports gave the acreage and product of each county. For a few years the yield per acre was given, but most of them have been computed by the writer. After computing the average yieldi ver acre for each county for each year from 1887 to 1895 , the average yield fer acre for the nine years for each county was found, which with the minimum and maximum yield per acre during that period is given for cach county in Table 2 below. These figures formed a very accurate means of determining the actual average crop of each county prior to the advent of the weevil. It remained to secure the acreage for each county since 1899, the acreage of 1899 being given by the Twelfth United States Census and probably being the most accurate obtainable upon which to base estimates of subsequent acreage. 


\section{THE COTTON ACREAGE OF TEXAS.}

Upon application to the Bureau of Statisties of the United States Department of Agriculture in 1903, we were inforined that no statistics of acrenge by counties were availahle. Other soures from whom estimates of any reliability could be sccurci were therefore sought. Fortunately the twn lea!ling newspapers of the State, tl a Dallas-Galveston Nows and Houston Posi, rave for the past few years gone to consideraible expense in collecting and publishing tine reports of from 300 to 500 zeporters for each paper, upon the acreage, conlition, and yield of extrn end other crops during each season. Tlirough the courtesy of tile winagement of these papers the writer was riven access to their files. Wach report was scrutinized, and the average acreaga for each county, and the number of reporters, was tabulater as given by cach paper. The railroads of the State also make fresuent estimates of the acreage and probable crop of eotton alones their lires to air in prevaration for its movement, and through the courtesy of the officials of the Civlí, Colorado and Santa Fe, Houston and 'Texas Central, IIoustnn East and West Texas, San Antonı and Aransas Pasc, Missouri, Fimsas and Texas, St. Louis Southwestern and Fort Worth and Rio Grancie, we were furnished with copies of repurts of their agents as far as available. During 1903 and 1904 some 200 voluntary entomological observers scattered over the State reported to the writer as State Entomologist at frequent intervals upon the insect injuries of their vicinity and also upon the increase or decrease of cotton acreage, injury by weevil and probable crop. The number of observers and arerage report upon acreage for each county was likewise tabulated. $A$ circular letter was then prepared and over a thousand copies seut to county assessors, postmasters and railroad agents asking their opinion upon acreage for their counties. In 1903 the State Statistical Agent of the United States Department of Agriculture, Mr. Oswald Wilson of Fort Worth, commenced recording the reports upon erops by counties and schedules were prepared by him giving the acreage for each eounty, and upon these, each report was recorded and arranged by counties. Through the courtesy of Mr. Wilson we were allowed to summarize these reports and tabulated the number of reports and the average report upon acreage for each county for 1903 and 1904. Among these sourees of information there is but little choice as to accuracy and an estimate must be made from an arerage of all. The reports from all sources were therefore assembled upon a card for each county upon which was also given the product for each year as given by the census and the acreage of 1899 as a basis for subsequent acreage. The reports of the railroads mentioned above were given by stations. It was necessary to assemble these by counties and to then weight the report of each agent according to the proportion of the total crop of the county landled by his station, which was done with the aid of the tables on 
Slipments of Cotton by Rail in Bulletins 19 and 28, Division of Statistics, United States Department of Agriculture. The cards thus used were ruled as shown below for Brazos county.

\section{BRAZOS COUNTY.}

First infested, 1s!9. First injured, 1900. 19,213 acres. 22,069 bales. 0.44 bales per acre, 1894.

\begin{tabular}{|c|c|c|c|c|c|c|c|c|c|c|c|c|c|c|c|}
\hline \multirow{3}{*}{$\begin{array}{c}\text { Bales and hales } \\
\text { per acre. } \\
\text { Source. }\end{array}$} & \multicolumn{3}{|c|}{1900.} & \multicolumn{3}{|c|}{1901.} & \multicolumn{3}{|c|}{1902.} & \multicolumn{3}{|c|}{1903.} & \multicolumn{3}{|c|}{1804.} \\
\hline & & 473 & 0.41 & & $135 \overline{7}$ & 0.47 & 15. & .035 & 0.37 & 15 & 212 & .34 & 20,0 & .009 & .44 \\
\hline & $\vdots \frac{\dot{3}}{2}$ & 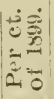 & $\stackrel{\dot{0}}{\stackrel{0}{0}}$ & 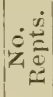 & 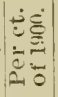 & $\dot{c}_{\pi}^{\infty}$ & $\dot{z} \frac{\dot{2}}{\frac{2}{2}}$ & 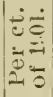 & $\stackrel{\dot{0}}{\dot{d}}$ & 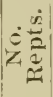 & 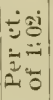 & $\frac{\dot{2}}{4}$ & $\dot{0} \frac{\dot{n}}{\vec{\pi}}$ & 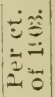 & 这 \\
\hline $\begin{array}{l}\text { Dallas News } \\
\text { Houstou Post... } \\
\text { Railroads } \\
\text { Observers, ludi- } \\
\text { viduals, ete... } \\
\text { U.S. Aids. } \\
\text { U. S. Schedule... }\end{array}$ & $\begin{array}{l}2 \\
4 \\
1\end{array}$ & $\begin{array}{r}! 5 \\
85 \\
100\end{array}$ & & $\begin{array}{l}4 \\
3\end{array}$ & $\begin{array}{c}100 \\
90 \\
\ldots \\
110\end{array}$ & & $\begin{array}{l}4 \\
3\end{array}$ & $\begin{array}{l}95 \\
9.5\end{array}$ & & $\begin{array}{l}4 \\
3 \\
4 \\
\frac{9}{2}\end{array}$ & $\left\{\begin{array}{r}8+5 \\
80 \\
80 \\
107 \\
6 .\end{array}\right.$ & $6 z$, , 1010 & 4 & $\begin{array}{l}115 \\
109\end{array}$ & \\
\hline $\begin{array}{l}\text { Combined esti- } \\
\text { mate. }\end{array}$ & 7 & 90 & 44,300 & 8 & 9. & $42,(85)$ & 8 & 95 & $\overline{40}, \overline{000}$ & 15 & 8 & 40,000 & ) 20 & 104 & 45,000 \\
\hline
\end{tabular}

On this card the number of bales at head of column for each year is that given by the census, while the bales per acre were estimater upon the number of acres as calculated at the foot of the column for that year. In most cases the weighed average of reports from all sources was taken; that is the number of reports from each source was multiplied by the average as reported, these added and the sum divided by the total number of reports. In many cases, however, from personal knowledge of the conditions in that county, or from apparent inacuracy of certain sources, they were disregarded in favor of uniform evidence from the majority of sources. The line giving "U. S. Aids" vives the summary of reports made to the State Statistical Agent, Tnited States Department of Agriculture as far as they could be classified by counties. The line "U. S. Schedule" gave the estimate oi acreage prepared by Mr. Wilson and used upon his schedules for making estimates, the total of which was practically the same as the 1) epartment's estimate of June, 1903. This was found in many cases to give a much larger acreage than any sources of information warranted. In such cases the highest figures reported for acreage of the county were usually used as the final estimate and often an additional acreage was added, to obviate any possibility of underestimating where thele was still a large difference between the two figures. This is only for 1903 as the schedules were prepared in that year. The basis of tive present acreage of the Department of Agriculture was therefore given careful consideration for each county, but this estimate of crunty acronges was merely for office use and n.st lop publication. Thus in Frazos county in 1903 all sources of information would have shown a marked decrease in acreage, 15 per cent, which would have given 34,000 acres based on the estimate of 40,000 for 1902. But as the De- 
pirtument's schedule gave 62,000 acres we made our final estimate of the acreage as 40,000 acres allowing $n$ r decrease from 1902 . In other words, though om estinates for 1900, 1901 and 1902 are most probably mirect for this county, and though there was undoubtedly all of 15 per cent decrease in 1903, in order not to by any means underestimate the acreage, in view of the Department's very large increase over 1899 , we practically added 6000 acres. This was done in numerous instances, in that our estimates of the total acreage of the State or of groups of counties are uniformly higher than the reports warranted, though rery much below the figures officially published by the Department. ihe total number of reports for cach county as far as collected for cach year was quite variable, but for the leading cotton counties was rmely under five and usually averaged between ten and fifteen.

Having illustrated the writer's method of. estimating the acreage it wili now be of interest to consider the acreage of the State as a wiole and of the different groups of counties from year to year and to compare them with the estimates from other sources, especially those made by the United States Department of Agriculture, which are the only official figures amnually published upon acreage.

Commeneing with 1899 the reports of the United States Census immst be taken as the ofticial figmres, for they are based upon an actual canvas of the territory and are the only published figures giving the acreage for each county. The Department of Agriculture has never published any estimates of the acreage of each county, and as far as the writer can ascertain never computed the acreage for each county in Texas until 1903, when estimates were made based upon the census ligures, and were tabulated for use in making future estimates, as previously mentioned. The report of the census showed 318,000 acres more in Texas and $: 54,000$ acres more in the Tnited States than the atimate of the Department of Agriculture.

Our estimate for 1900 shows $9 \% .9$ per cent of the acreage of 1899 or a decrease of 146,795 acres. For this year the Department of Agriculture gave an increase of 8 per cent. In the final estimate pitiblished by the Department (Bull. ¿8 Div. Statistics, see Table IV.), it is stated that the estimates have been revised "to bring them into reasonable conformity with those of the United States Census." To the Department's estimate of December, 1900, of 25,034,\%3t acres for Lnited States was evidently added 723,405 acres to give the $25,758,139$ acres for United States as finally published, which would represent very closely the difference between the census figures and those of the ]) epartment in 1899 . 
Boll Weevil and Cotton Crop of Texas.

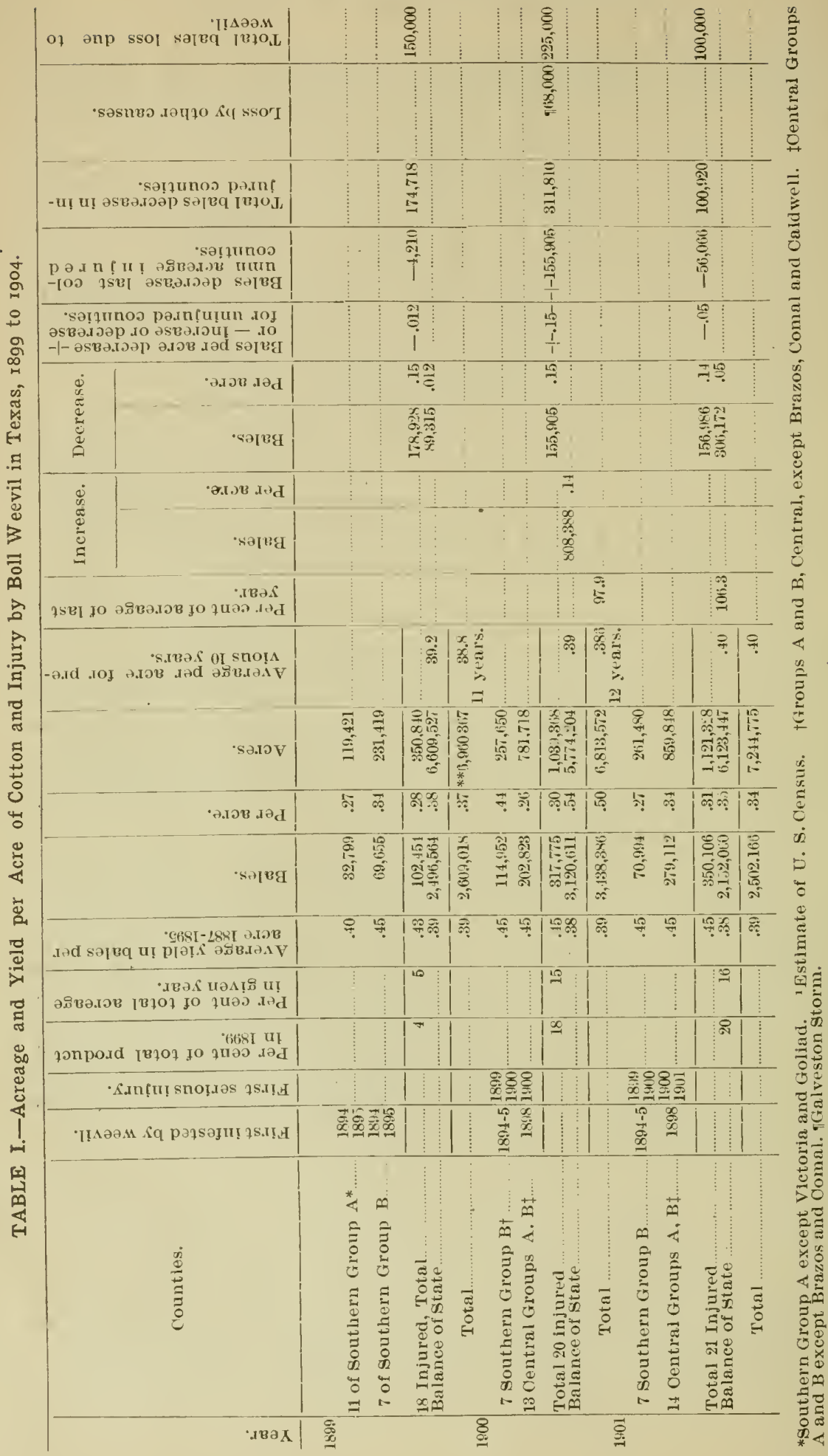




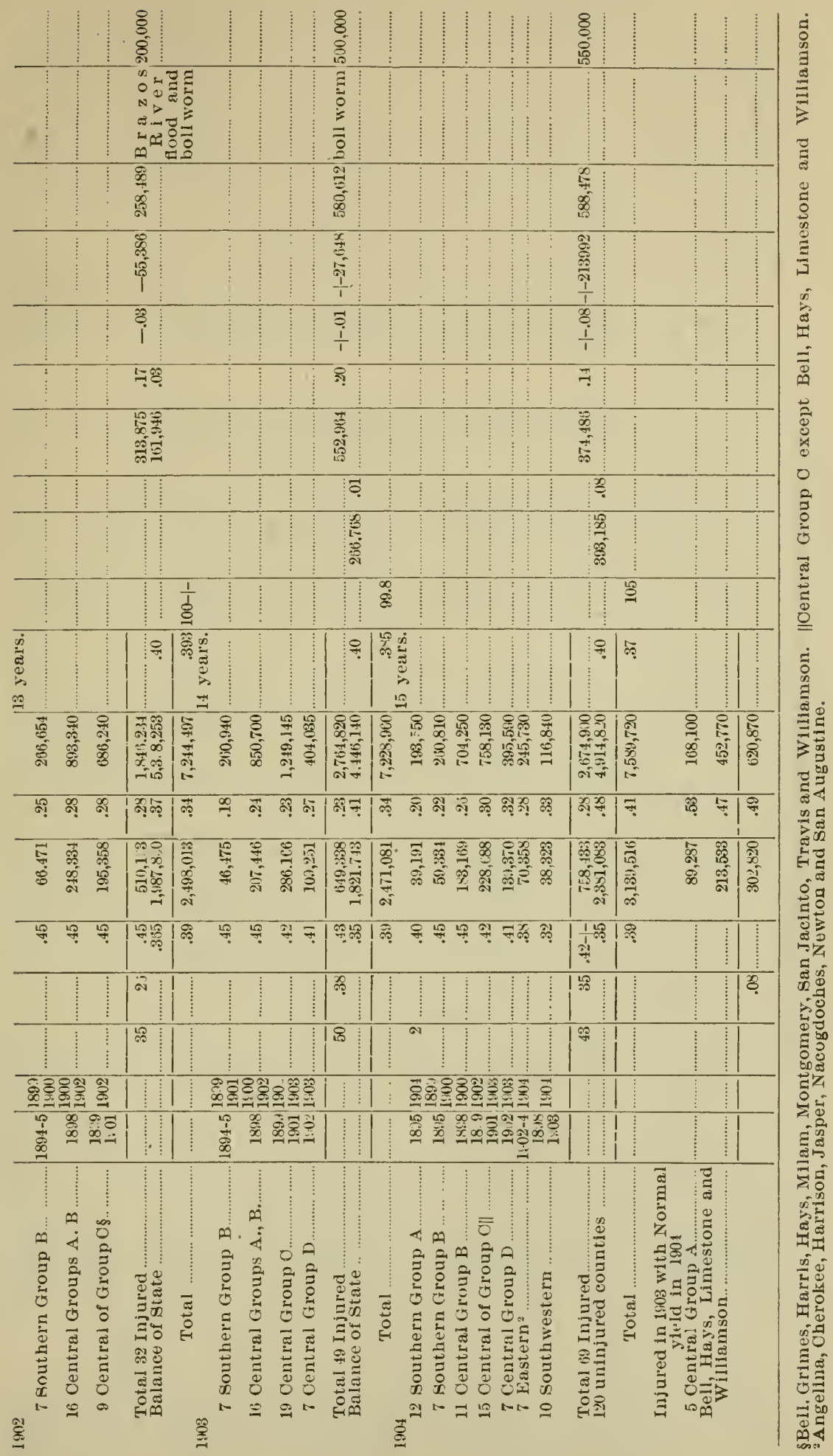




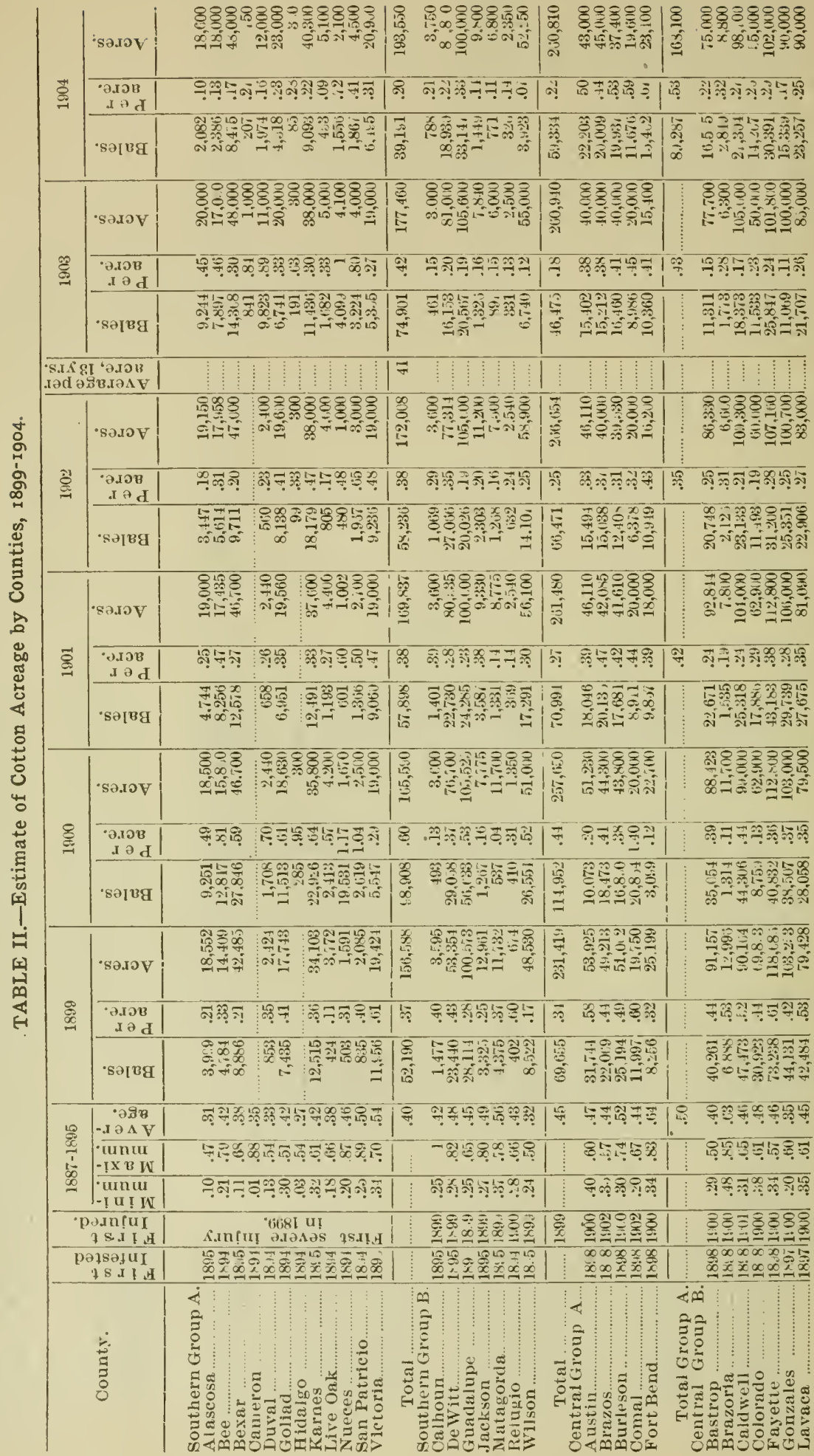




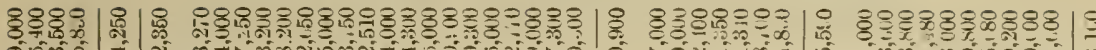

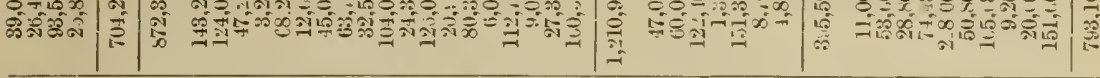

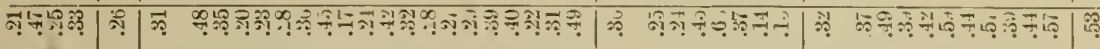

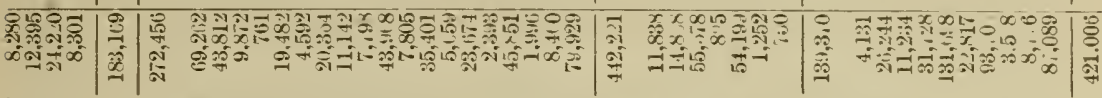

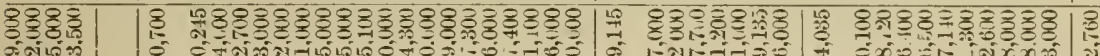
หล่าง

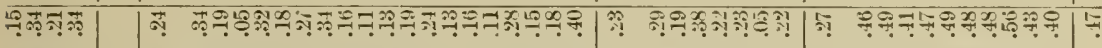

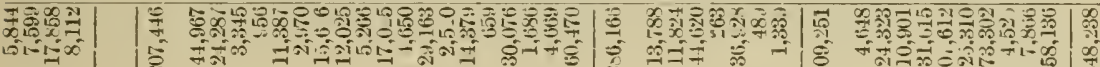
का

\begin{tabular}{|c|c|c|c|}
\hline 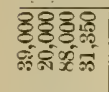 & 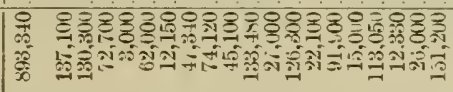 & 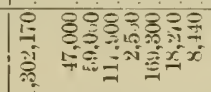 & 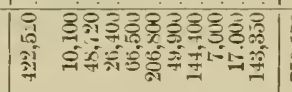 \\
\hline
\end{tabular}

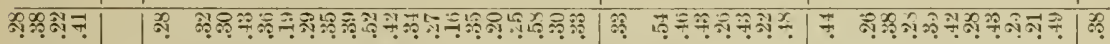

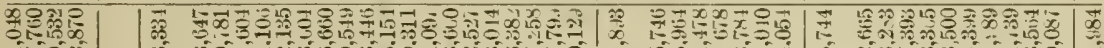
J-in| |

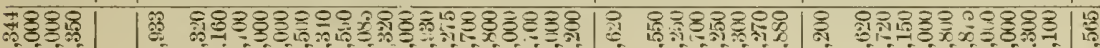

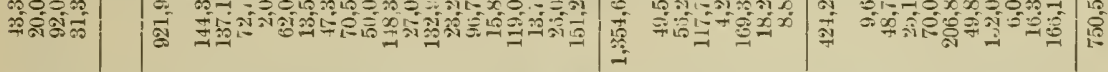

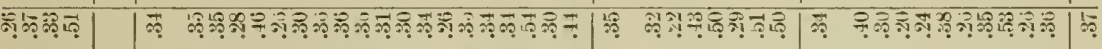

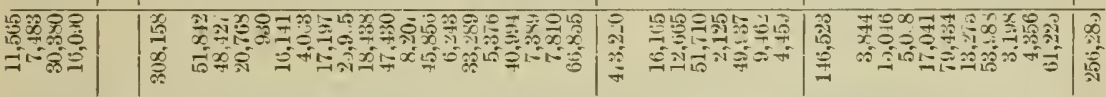

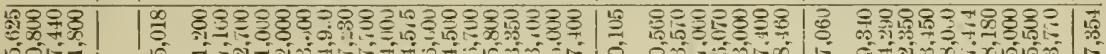

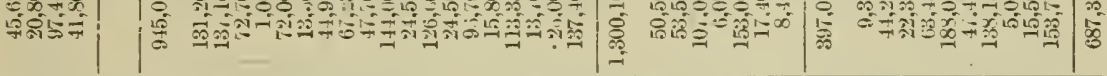

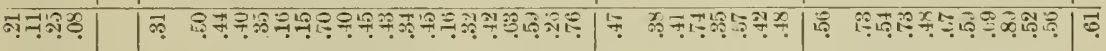

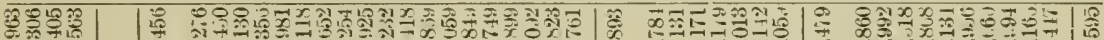
onon

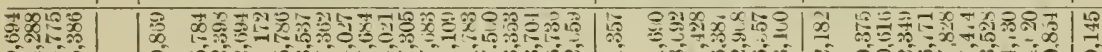

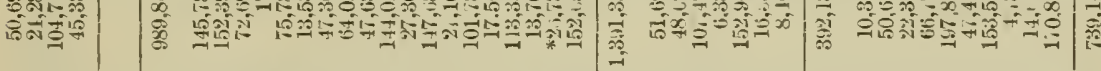

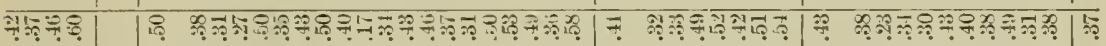

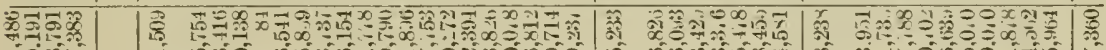

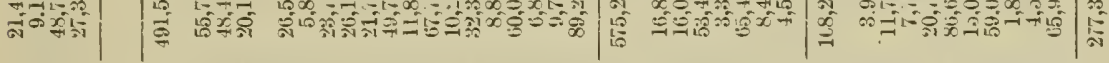

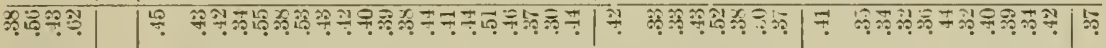

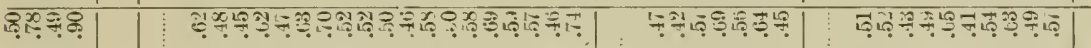

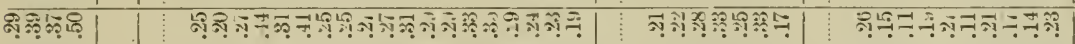

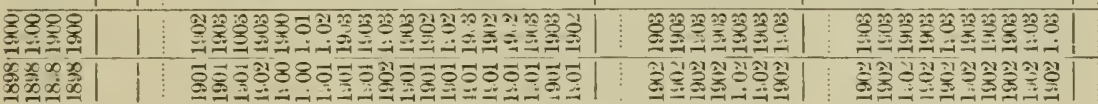

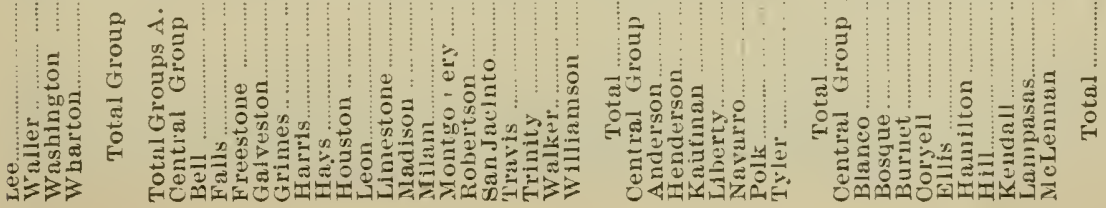


Boll Weevil and Cotton Crop of Texas.

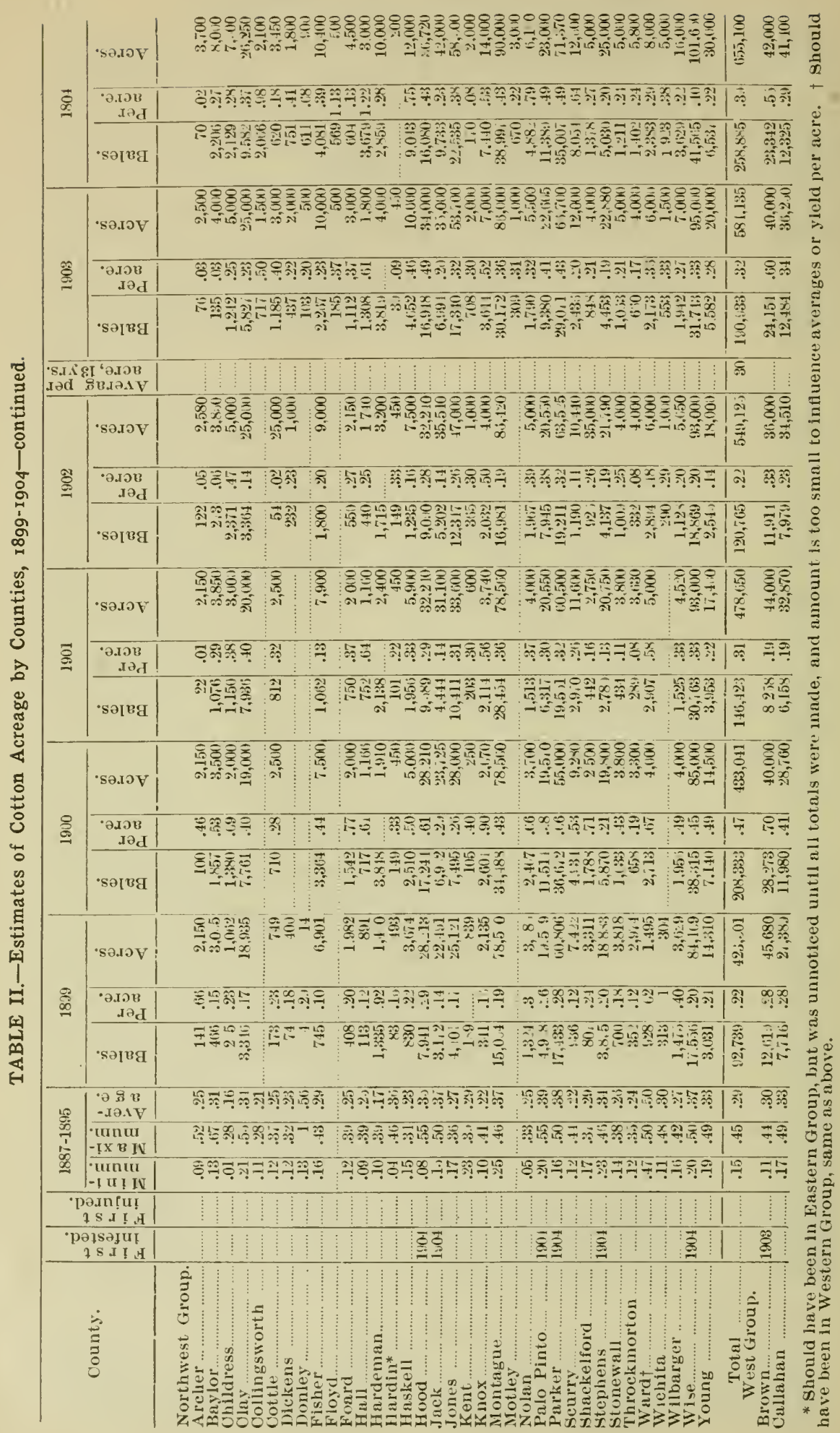


Boll Weevil and Cotton Crop of Texas.

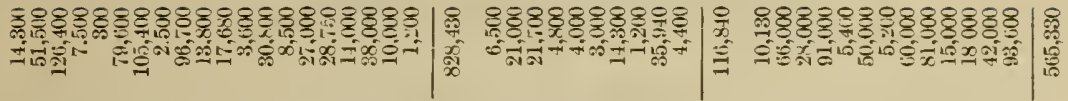

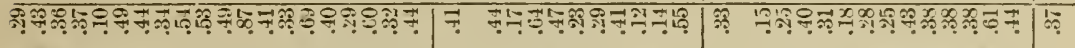

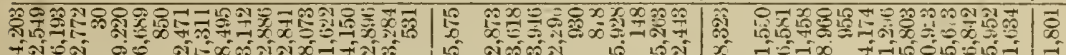

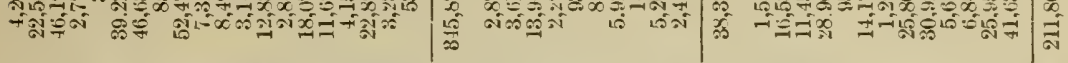

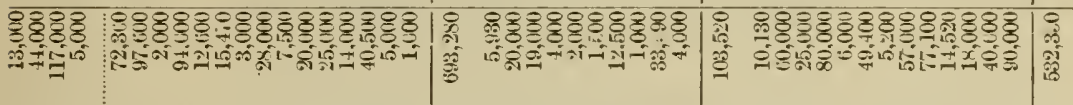

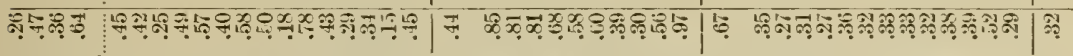

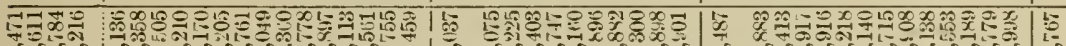

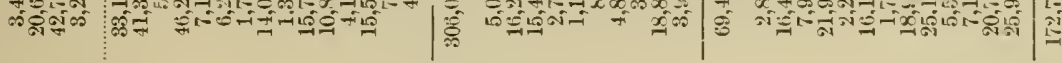

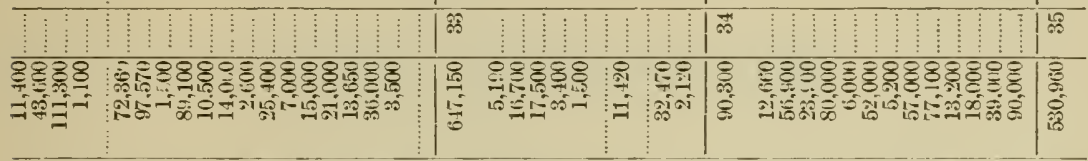

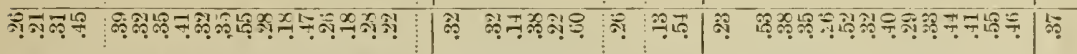

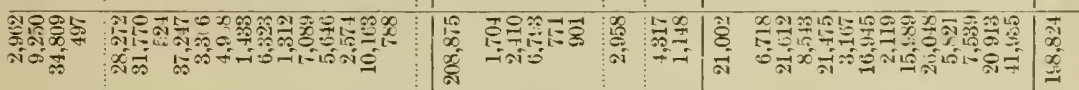

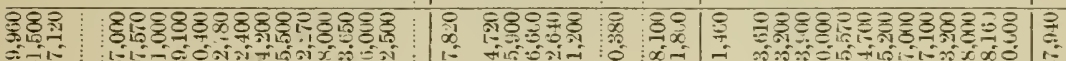

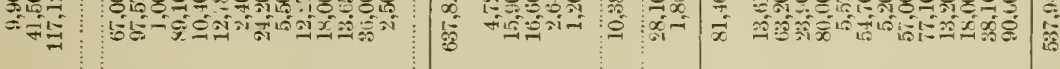

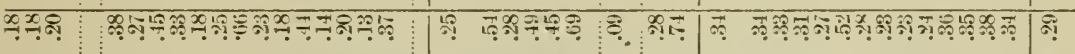

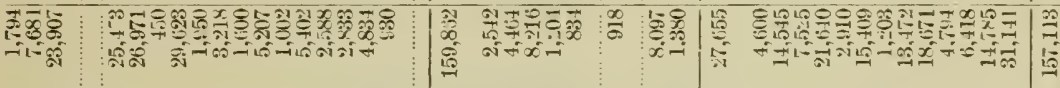

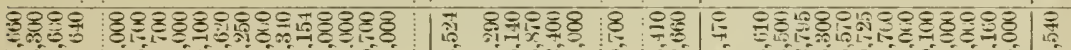

ऊิ๊ एक

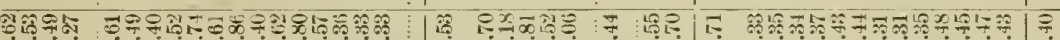

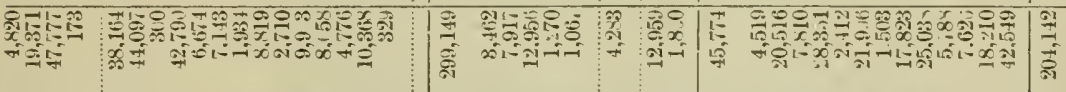

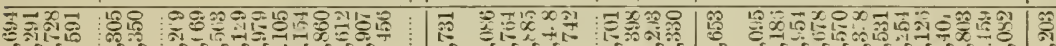

6ी

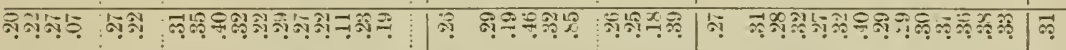

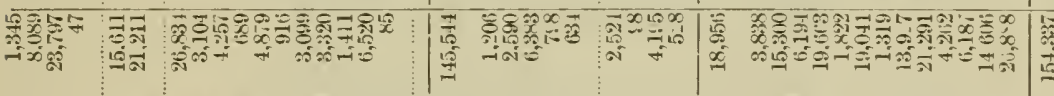

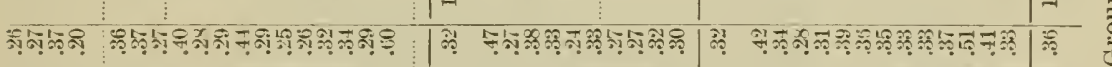

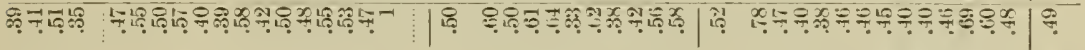

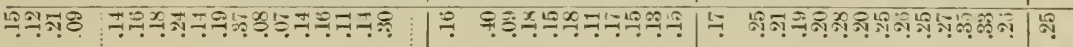

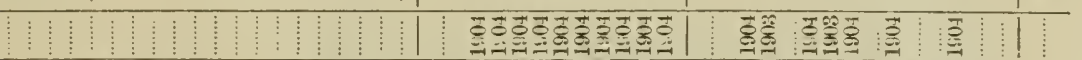

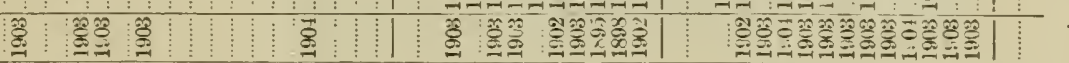

כ.

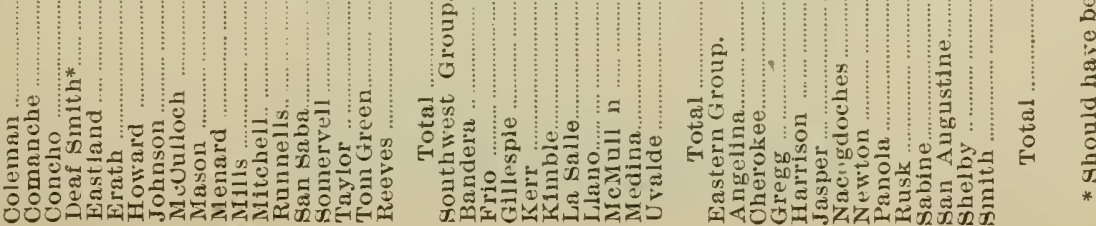


Boll Weevil and Cotton Crop of Texas.

\begin{tabular}{|c|c|c|c|}
\hline \multirow{3}{*}{$\Phi$} & 'sə.љे | & mong & 商 \\
\hline & . & 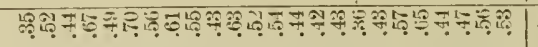 & $\vec{~}$ \\
\hline & 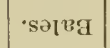 & 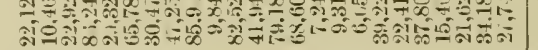 & 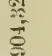 \\
\hline \multirow{3}{*}{ 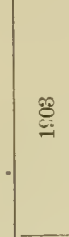 } & • & 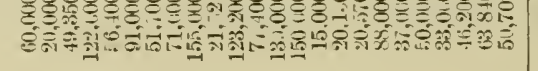 & 管 \\
\hline & 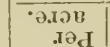 & 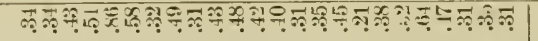 & $F$ \\
\hline & •sə[rg] & 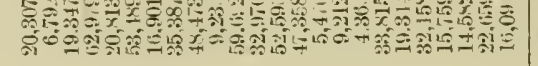 & 窝 \\
\hline \multicolumn{2}{|c|}{ 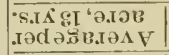 } & & $\neq$ \\
\hline \multirow{3}{*}{ 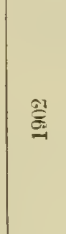 } & •sə.IวY & 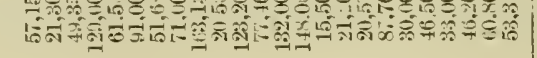 & 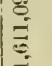 \\
\hline & 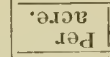 & 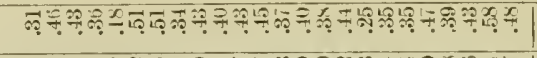 & 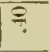 \\
\hline & •se[rg & 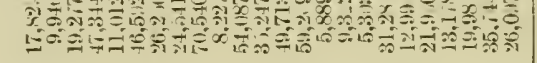 & $\frac{\sqrt{2}}{20}$ \\
\hline \multirow{3}{*}{$\overrightarrow{8}$} & 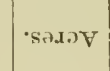 & 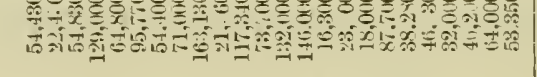 & 然 \\
\hline & 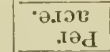 & 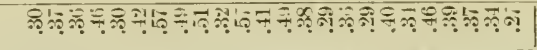 & $\stackrel{9}{\rightarrow}$ \\
\hline & "sə|rg & 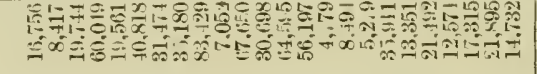 & 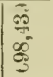 \\
\hline \multirow{3}{*}{$\stackrel{8}{8}$} & 'səגəV & 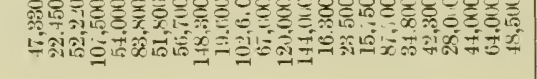 & 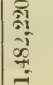 \\
\hline & 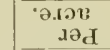 & 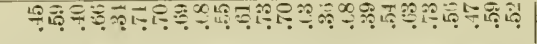 & $\bar{\square}$ \\
\hline & •sə[Bg & 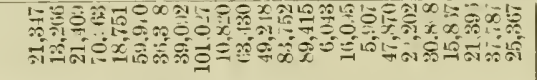 & 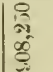 \\
\hline \multirow{3}{*}{ 茎 } & 'sa.tov & 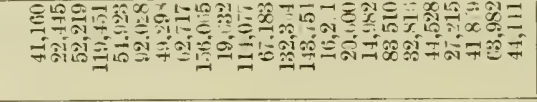 & 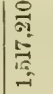 \\
\hline & 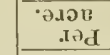 & 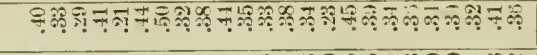 & ได \\
\hline & •sə[x] & 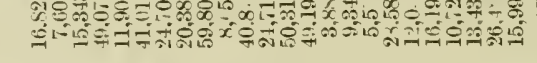 & 竞 \\
\hline \multirow{3}{*}{$\frac{\sqrt{8}}{\frac{1}{7}}$} & 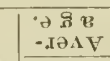 & 눅? & 承 \\
\hline & 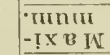 & 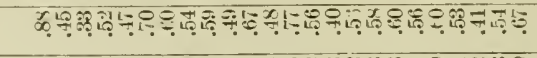 & 商 \\
\hline & 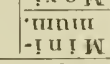 & 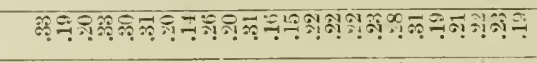 & | \\
\hline \multicolumn{2}{|r|}{ 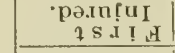 } & & \\
\hline \multicolumn{2}{|c|}{ 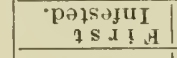 } & 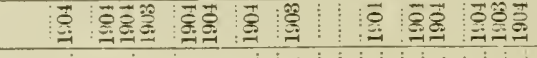 & \\
\hline \multicolumn{2}{|r|}{$\stackrel{\grave{\Xi}}{\grave{\Xi}}$} & 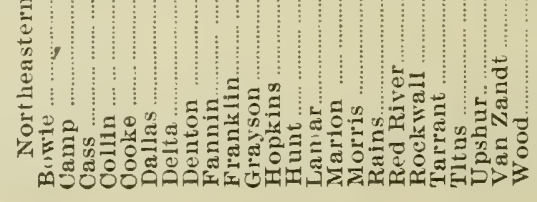 & 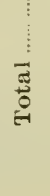 \\
\hline
\end{tabular}


Boll Weevil and Cotton Crop of Texas.

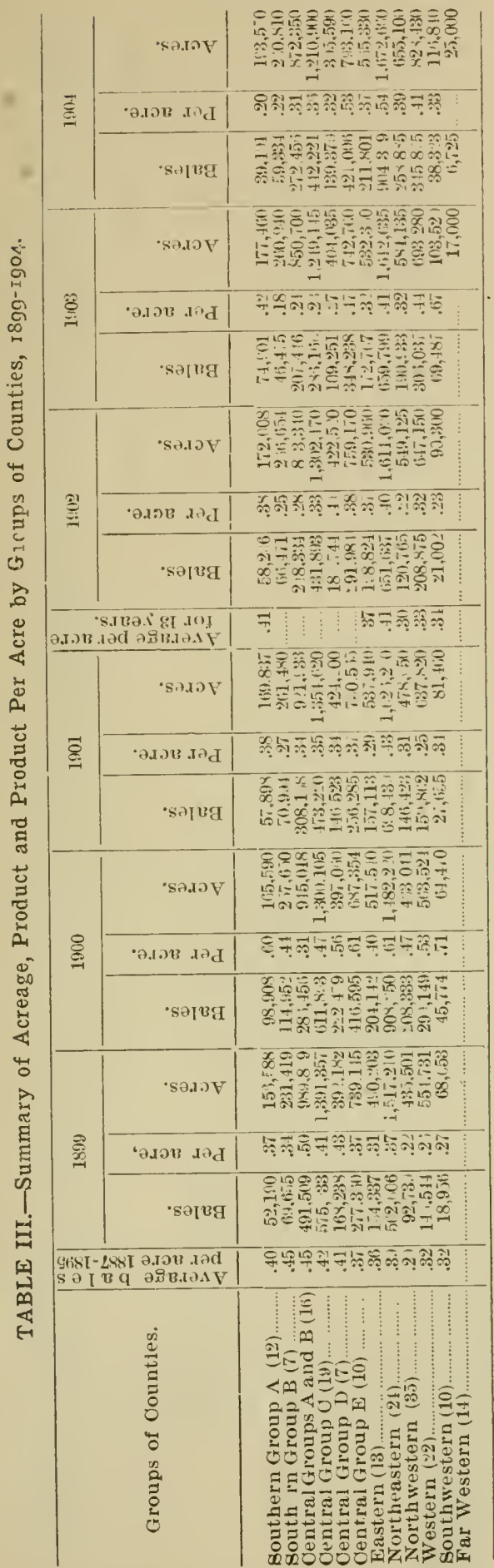


Boll Weevil and Cotton Crop of Texas.

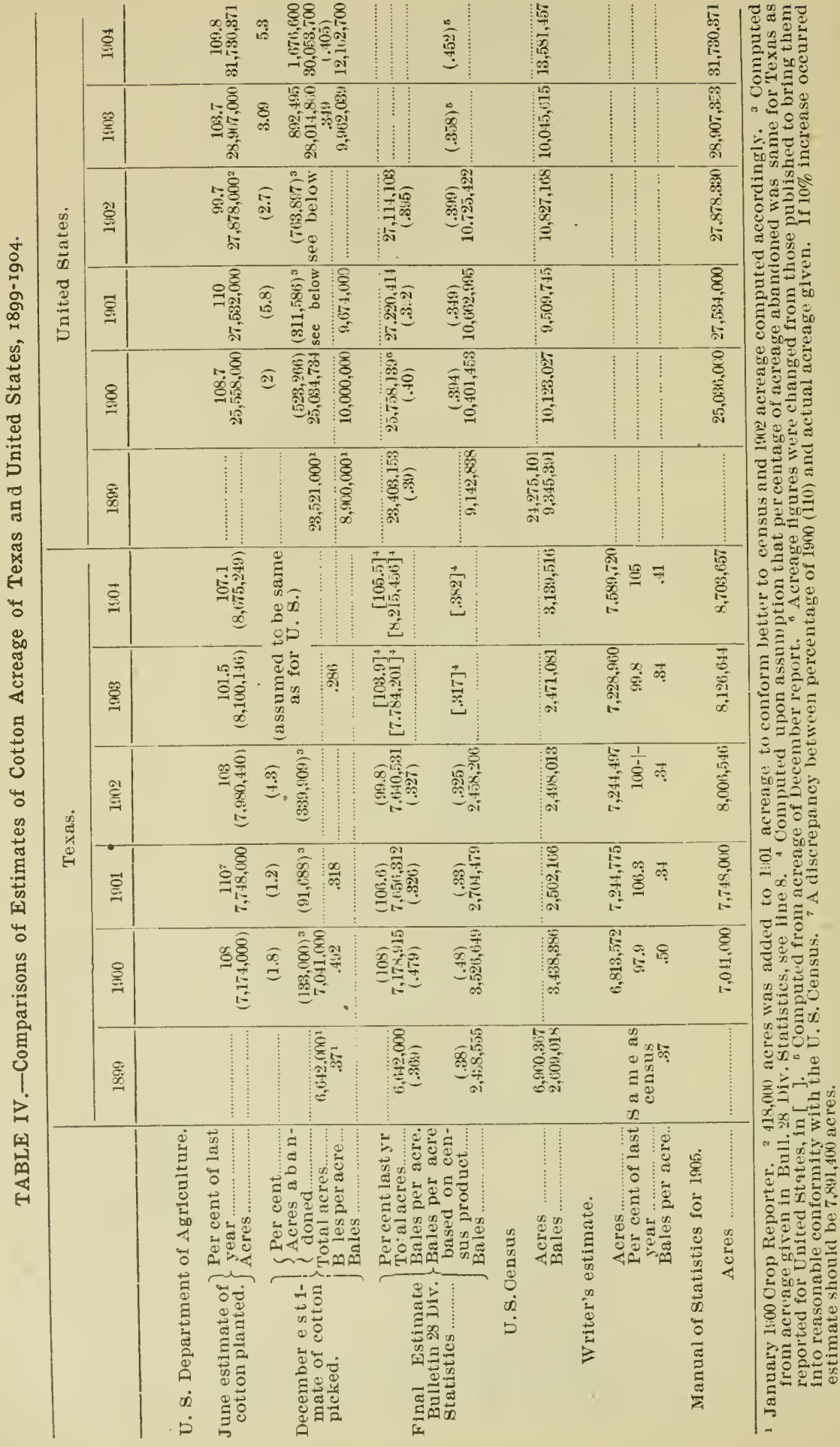




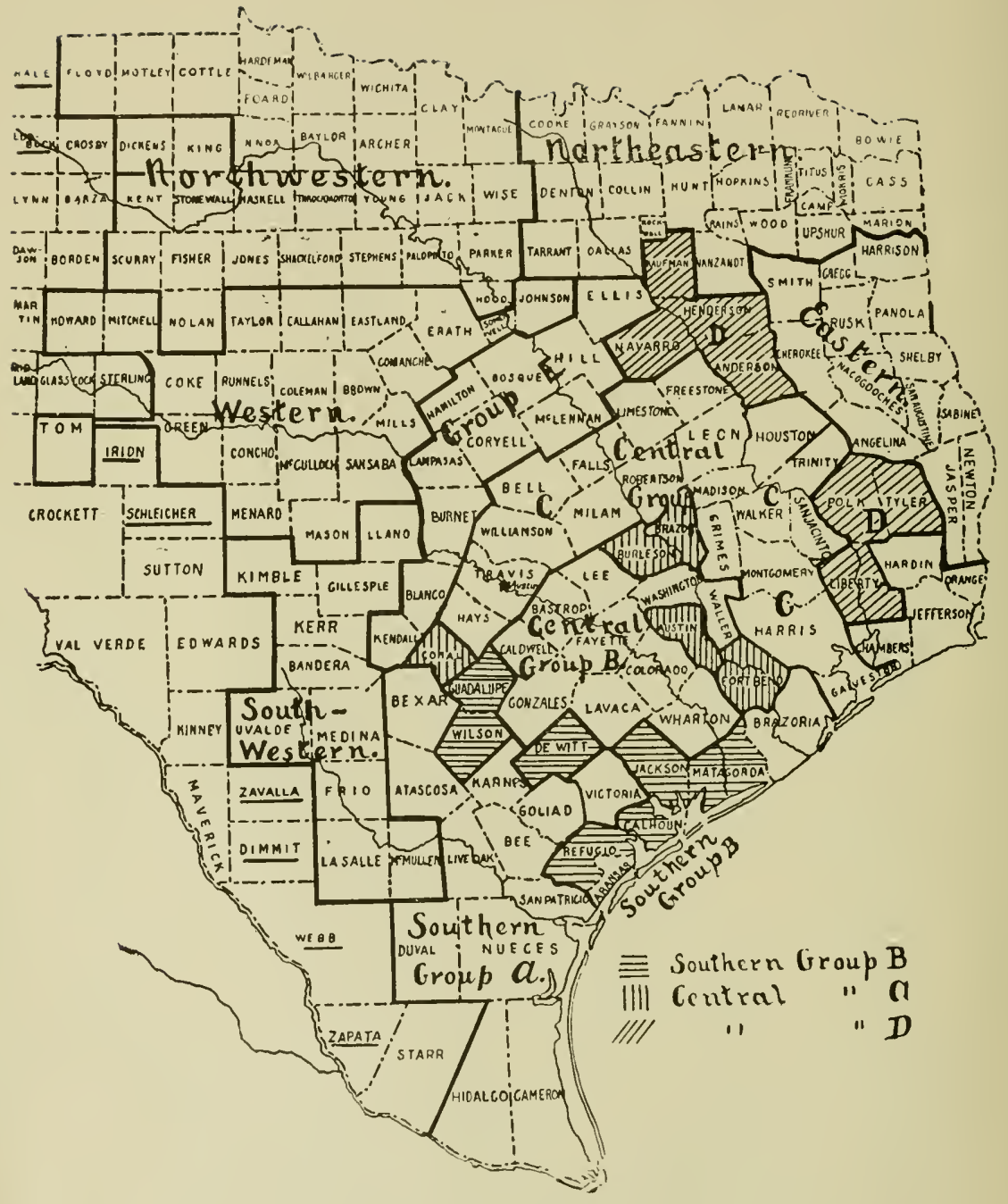




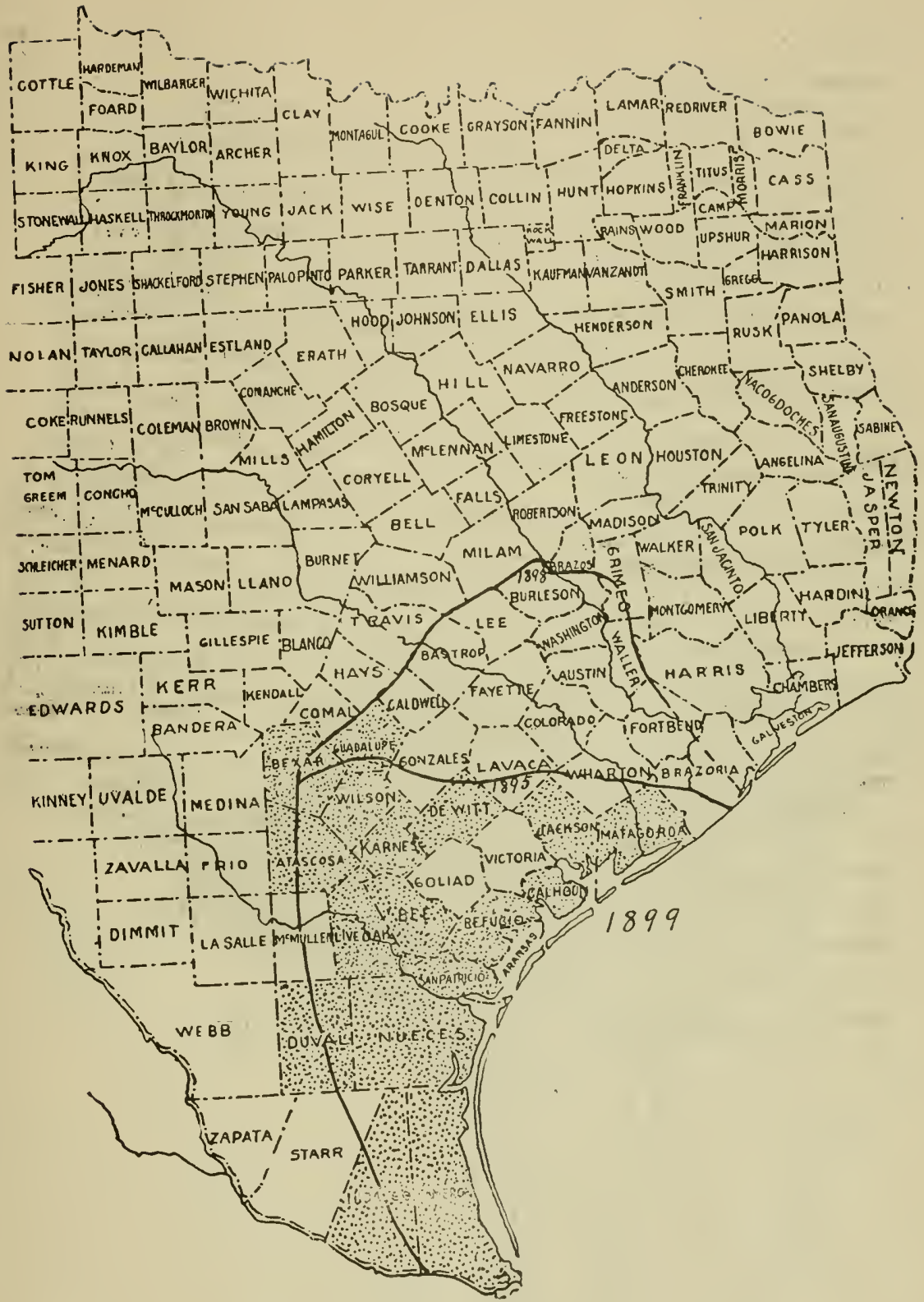




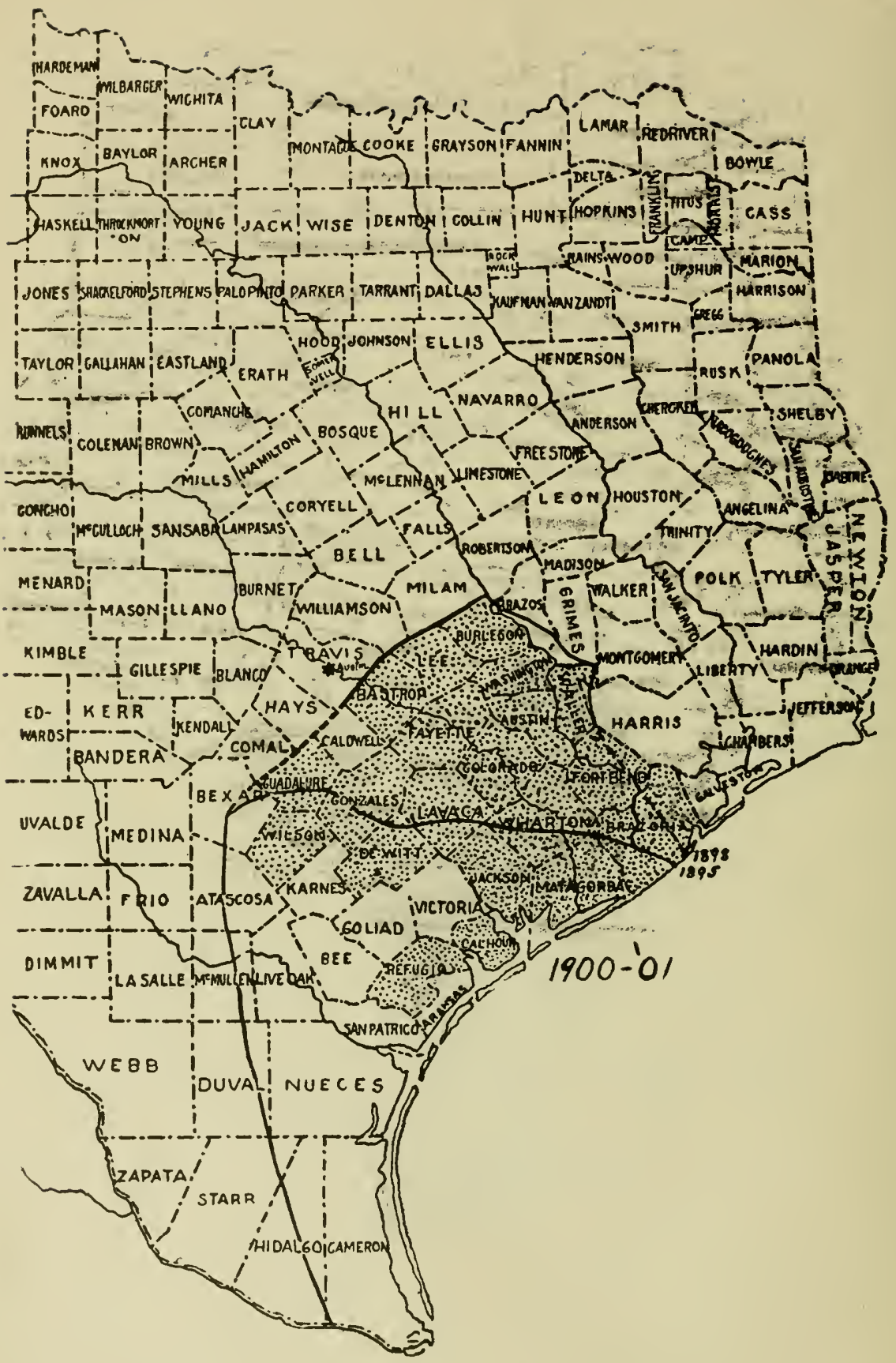




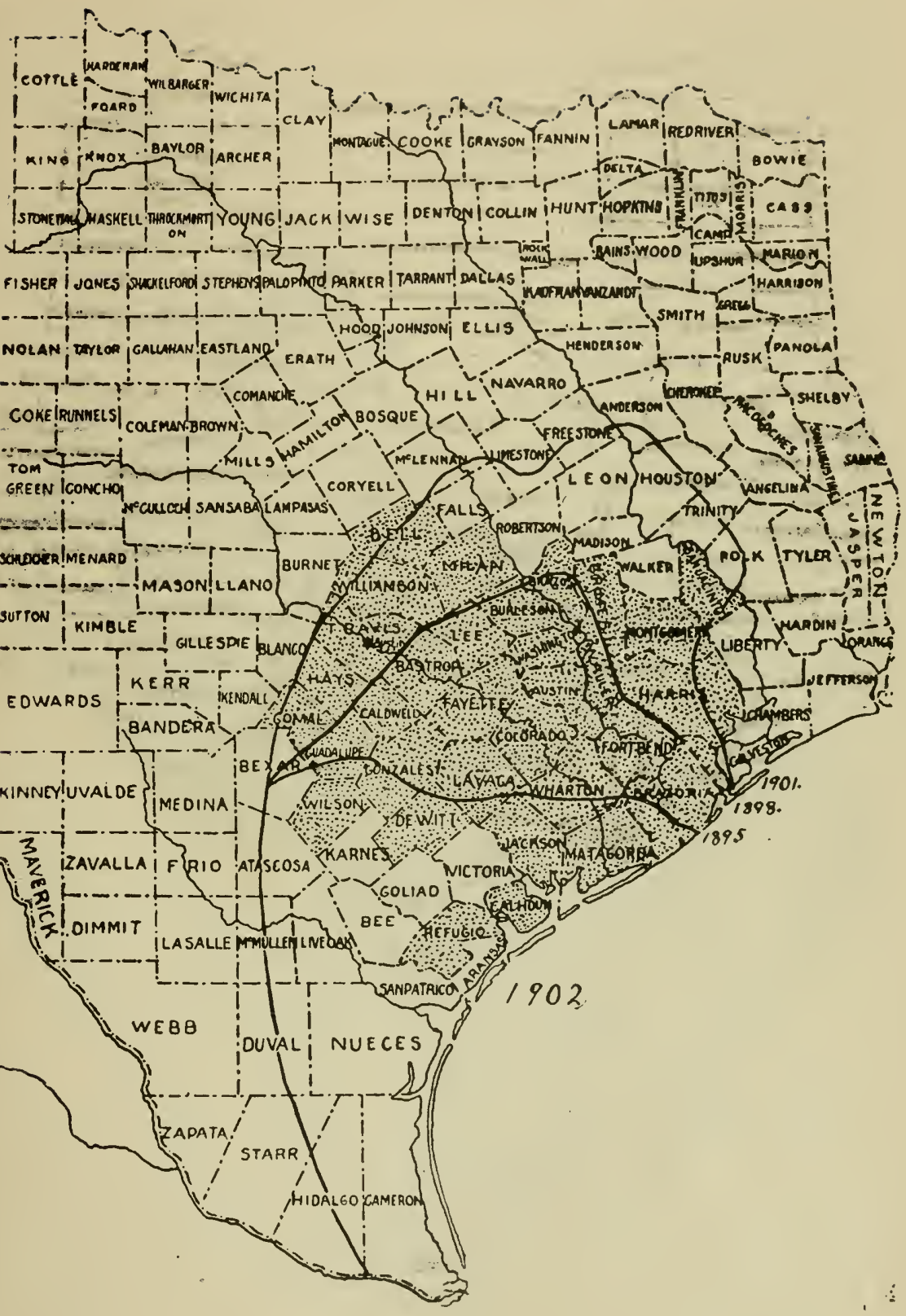




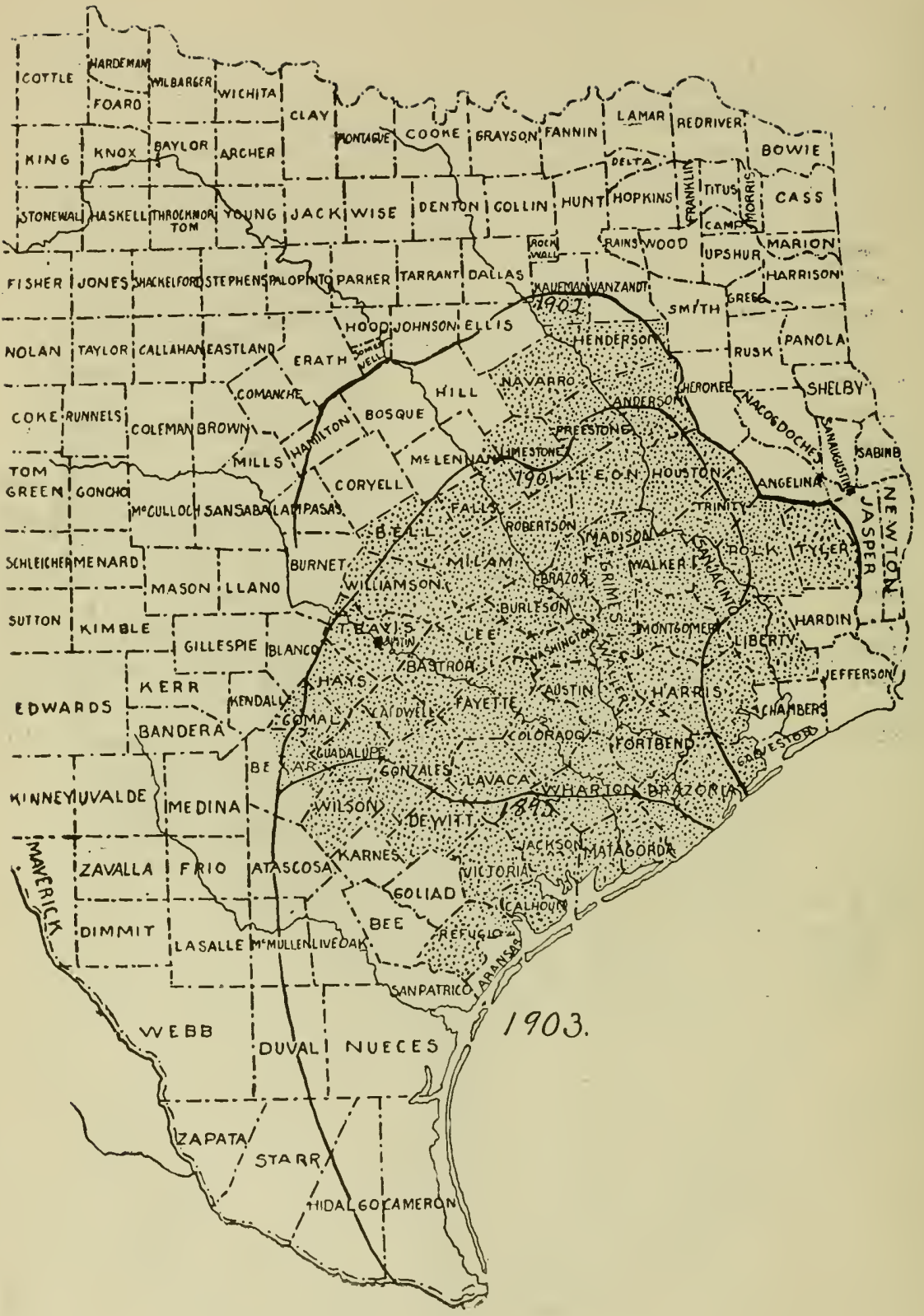




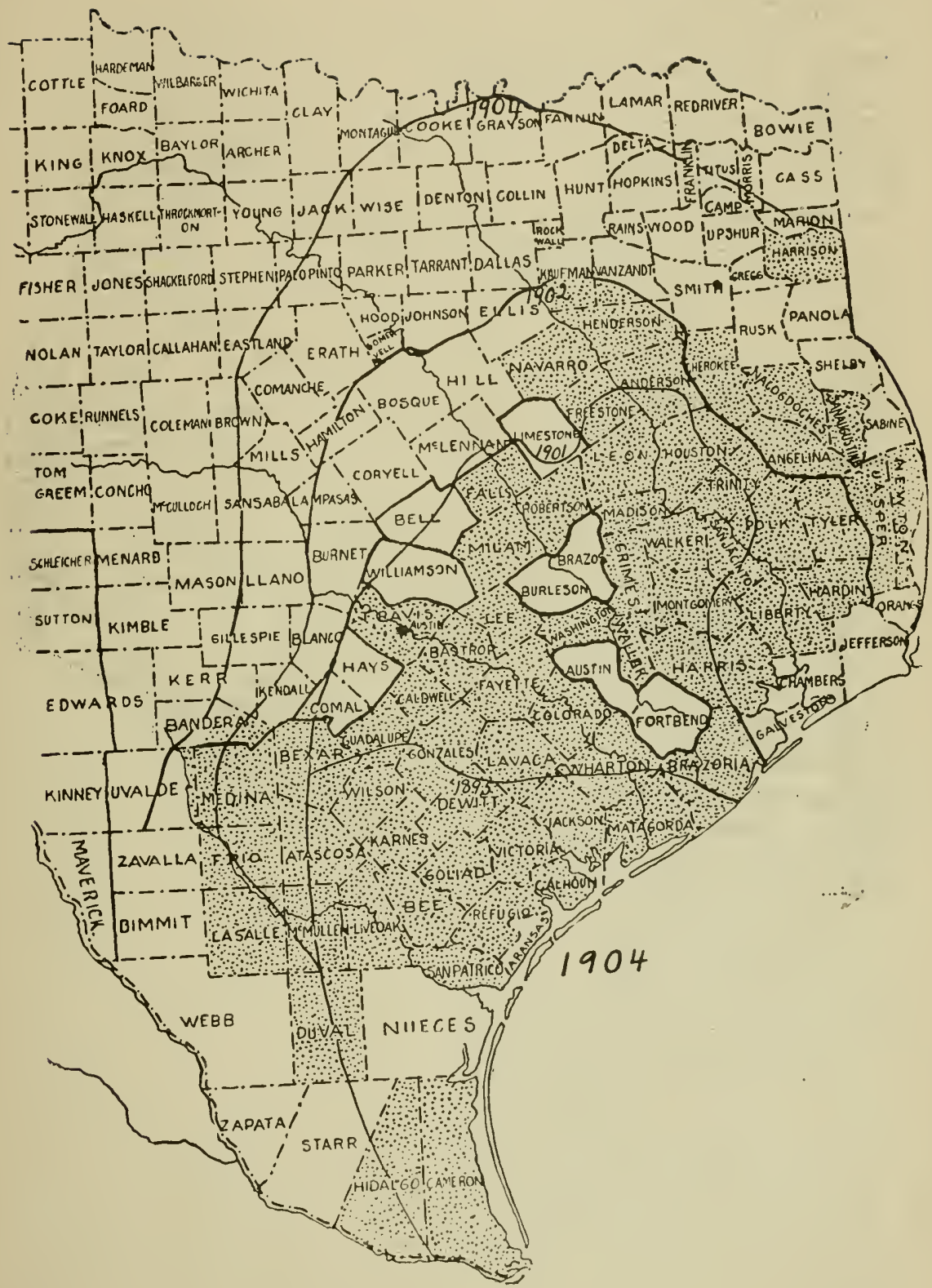




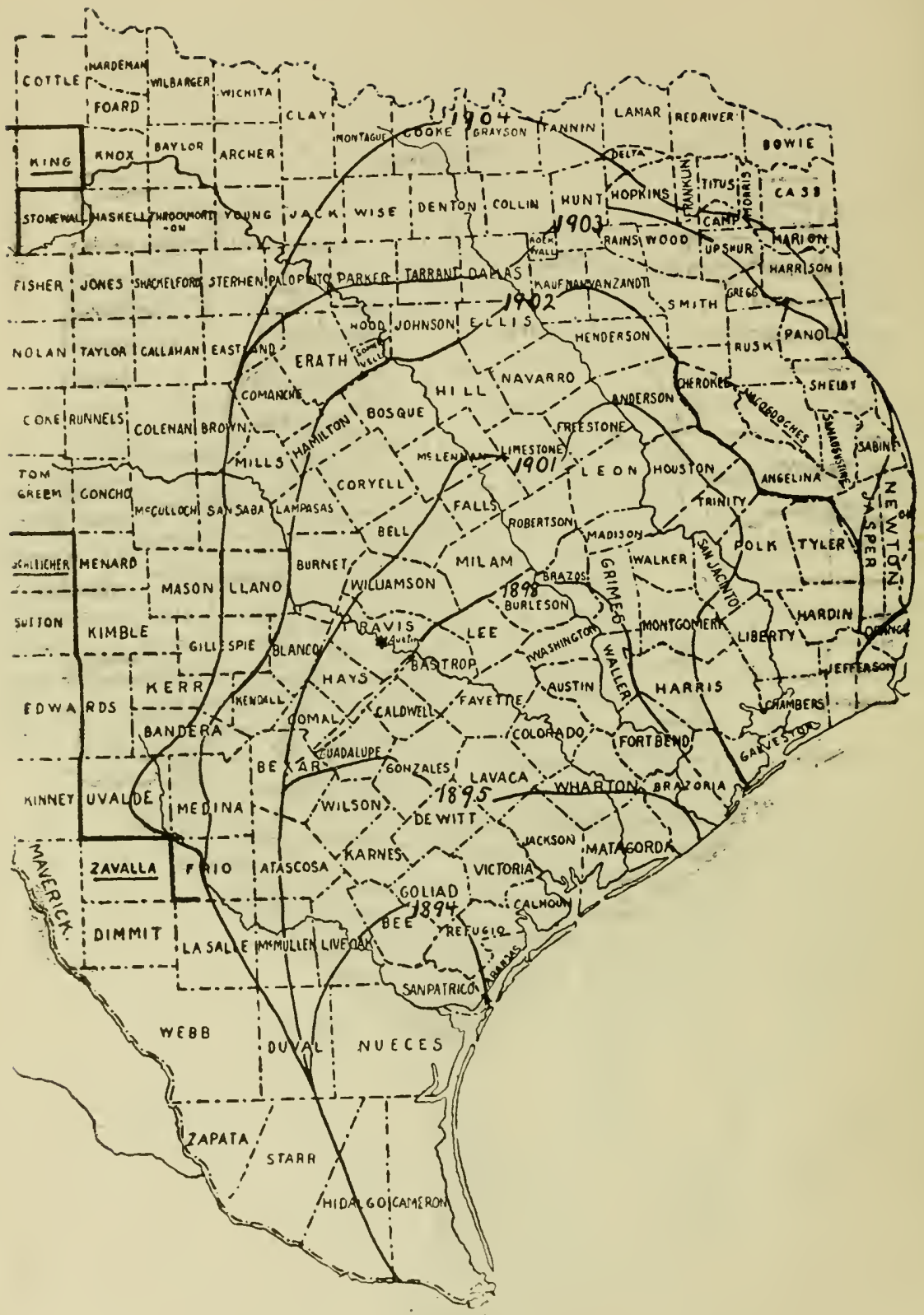




\section{TABLE V.}

COMPARISON OF ESTIMATES IN ACREAGE IN PERCENTAGE OF LAST YEAR.

\begin{tabular}{|c|c|c|c|c|c|c|c|}
\hline \multirow[b]{2}{*}{ Year. } & \multirow{2}{*}{$\begin{array}{c}\text { Galveston- } \\
\text { Dallas } \\
\text { News. }\end{array}$} & \multirow{2}{*}{$\begin{array}{c}\text { Houston } \\
\text { Post. }\end{array}$} & \multirow{2}{*}{$\begin{array}{l}\text { Ft. W. \& } \\
\text { R.G. R. R. }\end{array}$} & \multirow{2}{*}{ Writer's. } & \multicolumn{3}{|c|}{ U. S. Dept. of Agriculture. } \\
\hline & & & & & $\begin{array}{l}\text { State } \\
\text { Ager. }\end{array}$ & Planted. & Plcked. $†$ \\
\hline $\begin{array}{l}1900 \\
1901 \\
1402\end{array}$ & $\begin{array}{r}97.2 \\
107.1 \\
98.1\end{array}$ & siiglit & $\begin{array}{r}89.14 \\
128.52\end{array}$ & $\begin{array}{l}97.9 \\
106.3 \\
100 .\end{array}$ & & $\begin{array}{l}104 \\
110 \\
103\end{array}$ & $\begin{array}{l}(108) \\
(106.6) \\
(99.8)\end{array}$ \\
\hline 1903 & 91.4 & $\begin{array}{c}\text { decrease. } \\
105\end{array}$ & H. \& T. O. & 99.8 & 9.4. * & 101.5 & [108.9] \\
\hline 1904 & 109.32 & … & R. R. & 105 & $105.3^{*}$ & 107.1 & {$[105.5]$} \\
\hline
\end{tabular}

* Average of all reports coming to state statistical Agent.

+ Computed by writer as ohowi in lable $1 \mathrm{~V}$.

For Texas there was adled to the estimate of December, 1900, of acreage picked $13 \%, 915$ acres in making the final estimate of $\%, 178,915$, which represents 2 per cent of the 8 per cent increase. Without this addition, which would be the December, 1900, estimate of $\%, 041,000$ acres, the Department would have shown an increase of but 1.1 per cent over the acreage of the census for 1899, though 6 per cent over its own estimate for 1899. These final estimates of the Department were not published, howerer, until June, 1904.

From a careful study of all the reports available we fail to find any reason for estimating an increased acreage in Texas in 1900. In addition to the reports of the News and Post, the writer has used in his estimate for this year reports from all agents of the Missouri, Tiansas and Texas Railroad, Cotton Belt and Fort Worth and Rio Grande Railroad. The report of the latter road shows a marked decroase for Northwest Texas and both of the papers agree in a decided flecrease. During the summer of 1899 the Brazos valley suffered from two unparalleled floods, which, according to investigation of the Department of Agriculture (Circular 10 Div. Statistics), damaged 339,000 acres of cotton. Undoubtedly a large portion of this acreage was unplanted in 1900 and our estimate shows a decrease of $17 \%, 894$ acres in Central Groups A, B, C, and E in 1900. In addition the Northeastern-the largest-Northwestern and Southwestern groups showed a decrease while the increase in the remainder of the State amounted to but 76.241 acres. A difference of 365,343 acres arises between our estimates and those of the Department for 1900 .

In 1901 we estimate an increase of 6.3 per cent over 1900 , being but 3-10 of one per cent less than that given for the Department in its final acreage picked. It may be well to here call attention to the difference in the estimates of the Department of Agriculture given out in June and December and the final estimate. The June estimate is of acres planted and is based on schedules returned the last week in May. It gives the percentage of the area planted the previous year for each State and the United States and an estimate of the total acres 
for the United States. In 1901 only was an estimate published for each State, and the June estimate of the previous year given in acres. (See Table IV.) In December an estimate has usually been published of the number of abandoned acres, the percentage abandoned of acres planted, and the number of acres picked and to be picked. This was given by States in 1900, but since then only for the total United States. The final estimates of acreage for 1899 to 1902 were published in June, 1904, and though no statement is so made, doubtless represent the acreage picked, as they are uniformly less than the June estimates, except for 1900 accounted for above. Since 1902 no final estimates have been published and the final acreage picked in Texas can only be estimated for Texas as in Table IV, by assuming the same percentage abandoned in it as for the total United States. The difference in these estimates is important in any statistics dealing with the vield per acre of the cotton crop. For instance the Manual of Statistics and Stock Exchange Handbook for 1905 gives the acreage as shown in Table IV, crediting it to the estimates of the United States Department of Agriculture. Evidently the June estimates wore used and those for Texas for 1902, 1903, and 1904 somewhat modified. If these figures based on the Department's June estimate are used the yield per acre will be much smaller than that later published loy the Department, and indeed than should be fairly used. The reports from which the writer's estimates are, made were usually made the second week in June, though some later, even into July, except in 1903 and 1004 , in which years the reports of the aids of the State Statistical Agent of the Department of Agriculture made May 25 viere also msed. Our estimates should, therefore, be somewhat less than those of June by the Department, and might be slightly more than its December estimate, but are found to be lower than the latter in 1900 and 1903 and closely approximating the final estimate (line \%, Table IV) of increased acreage over the final estimate of the previous year as computed in Table IV. In 1901 an increase was shown in all groups of counties except Central Group AB, which was seriously injured by the Gaiveston storm and boll weevil in 1900 .

The total acreage for 1902 is estimated as the same as that for 1901. Southern Groups A and B, Central Group E, the Northwestern and Southwestern counties, showed some increase, while the ialance of the State showed an equal decrease. The June estimate of the Department gives an increase of 3 per cent over 1901. In the June Crop Ficporter for 1902 it is stated that the acreage estimated then for the Cinited States was based upon that of 1901 plus 418.000 acres added to make it better agree with the census report (published in 1902). Of-the discrepaney between the acreage of the Census and Departinent in 1899, the Texas acreage comprised 42 per cent. If this 418,000 acres added to 1901 before estimating 1902 were evenly distributed between the States according to the actual discrepancy of 1899 , then 175,560 acres would have been added to Texas, which would represent 2.3 per cent of the increase estimated for that year, leaving the increase at 0.7 per cent, but little above that of the writer. The final estimate 
of acreage for this year as given by the Department in 1904 was but 99.8 per cent of 1901, practically the same as our estimate.

The acreage continued to remain practically the same in 1903 , being 99.8 pèr cent of 1902 . The summer of 1902 witnessed another serious flood of the Brazos and an increased area injured by the bol! weevil, which resulted in a continuance of the decrease of acreage in the central counties. The Central groups and Southern Group B showed a decrease of 4.3 per cent from 1902, while the balance of the State showed an increase of 2.5 per cent. In addition to the News and Post, reports from the Houston East and West Texas Railroad, Houston and Texas Central Railroad and Fort Worth and Rio Grande Railroad, were consulted, reports being given from each agent of these roads. The arerage of 584 reports received by the State Statistical Agent of the United States Department of Agriculture from railroads, oil mills, and aids, was practically 94 per cent of the 1902 acreage. No final report has yet been made by the Department.

Assuming the abandoned acreage to have been the same as for the United States, the picked acreage would have been 3.9 per cent greater than the final acreage of 1902 (See Table IV, line 7 ), but probably the abandoned acreage in Texas was above the average, and it is pnssible that the final figures of the Department will show but little increase over the acreage picked in 1902.

The high prices of the fall of 1903 induced a considerable increase of acreage in 1904 in many parts of the State, making a total of $360, \% 60$ acres or 5 per cent above that of 1903 . Central frouns $C$ and $D^{\prime}$ which had been very seriously injured by the boll weevil and 1.oll worm in 1903 decreased their acreage 2.2 per cent. The remainter of the State increased; Central Group AB, 2.4 per cent, Soutliem Group B about the same, the Southwestern, Southern A, Eastern and Northeastern a total of 4.6 per cent, while the Northwestern and Western increased 16 per cent over 1903 . The June estimate of the Department of Agriculture gave an increase of $\% .1$ per cent. If the per sent uf the planted acreage abandoned in Texas in 1903 and 1904 be considered the same as for the total United States, then the picked acreage for 1904 , computed upon this basis, would be but 5.5 per cent greater than the picked acreage so computed for 1903 . Our estimate agrees almost exactly with the average of the reports made to the State Statistical Agent, which was 5.3 per cent increase. This includes, however, reports not arranged by counties from oil mills and railroads and recognized no decrease in any section of the State, where we have incicated a slight decrease in Central Groups $\mathrm{C}$ and $\mathrm{D}$, almost exactly amouniting to the difference in the two estimates of 0.3 per cent. Our estimates included reports from all agents of Gulf, Colorado and Santa Fe Railroad, Fort Worth and Rio Grande Railroad and estimate of Houston and Texas Central Railroad.

Comparing the total acreage of 1904 with that given by the census for 1899 , there is an increase of 9 per cent for the State. This statement is, however, quite misleading. The northwestern and western counties increased 51 per cent in this time and all of the counties un- 
injured by the boll weevil in 1903 (140) increased 23 per cent, while the 40 eounties injured by the boll weevil in 1903, in 1904 had decreased over 7 per cent from 1899 and comprised 41 per cent of the total acreage of the State.

Against this the June, 1904, estimate of the Department of Agriculture would show an increase of 28 per cent over 1899 and the computed picked acreage for 1904 would be an increase of 22 per cent over 1899. The June estimate of the Department for 1904 is 1,085,529 acres more than that of the writer and the computed picked acreage based upon this is 625,736 acres more. It would seem that the Department's June estimate is probably 700,000 or 800,000 acres too large, and that the acreage picked computed on the basis of the June estimate and percentage abandoned is at least 500,000 acres too large since June, 1903. Undoubtedly this is to be mostly accounted for by a failure to recognize the decreased acreage of the counties badly injured by the boll weevil and floods in Central Texas, as well as to a lack of sufficiently careful analysis of reported increases from different parts of the State.

Several considerations support this view that the 'Texas acreage has been heretofore orerestimated. A brief glance at the development of the acreage in Texas for the past 25 years as compared with the rest of the cotton belt may not be inopportune. From 1879 to 1889 the cotton acreage of 'T'exas increased 80.61 per cent, while in the States east of the Ilississippi River and Louisiana it increased an average of 46 per cent. From 1889 to 1899 the Texas acreage of cotton increased ry per cent, practically the same as the previous decade. The Twelfth Census shows a total iecrease of "improved" farm land in Texas due to a difference of classification, but if we assume that the proportion of "improved" farm land to the total farm area was the same for the previous censuses, it would seem that the increase of cotton acreage had almost exactly equaled the percentage of increase of "improved" farm land, or in other words, that the increase of acreage in cotton was cine to the opening to settlement of new farms. From 1889 to 1895 the Texas acreage increased 48 per cent while the rest of the cotton States declined 11.6 per cent. From 1895 to 1899 Texas increased 14 per cent, while the rest of the cotton States increased 16.8 per cent, but not including Indian Territory and Oklahoma, they showed 14 per cent increase, the same as Texas. The cotton States, exclusive of Texas, Oklahoma and Indian Territory, decreased their cotton acreage 1.9 per cent from 1889 to 1899 according to the estimates of the United States Department of Agriculture, whereas the census gives an increase of slightly less than 2 per cent. In 1899 Texas had 35.6 per cent of her improved farm land in cotton, or 45.7 per cent of all crop acreage. At the same time practically the same ratio maintained in the largest cotton States of Alabama, Georgia, Mississippi and South Carolina. Thus in 1899 Texas had been increasing her. cotton acreage at the same rate as the balance of the cotton belt for five years and had practically the same proportion of her farm acreage in eotton. From 1899 to 1904 the Department estimates would show an increase of 44 per cent in the acreage for the cotton belt exclusive of Texas, and 22 to 28 per cent for Texas. We estimate the increase for Texas at 9 per cent. Since 1899 
Texas has been subject to many conditions affecting the cotton industry which have either been unknown or very much less important in other cotton States. During this time the press of the State and her agrieultural leaders have night and day preached "diversification." "That inis has been heeded all Texans are aware. Trucking has become an increasingly important industry all through eastern, central and southern Texas. East Texas has seen a marvelous development of the firuit industry. The cotton acreage in the coast counties has undoubtedly been diminished by the rice and oil industries. An unprecedented flood of the Brazos in 18.9, another in 1902, and the terrible storm of September, 1900, all tended to diminish the cotton acreage in some of the most productive counties. In addition to all these factors making for the reduction of cotton acreage, the boll weevil has spread steadily north and castward until it now injures fully half the acreage of the State and destroys practically one-half the crop in this territory. It is certainly unreasonable to suppose that, in the face of a constantly diminishing yield per acre, planters in the injured district will continue to increase their acreage, though this has undoubtedly taken place to some extent in single seasons.

Notwithstanding these facts the counties minjured by the boll weevil have increased 23 per cent from 1899 against 14 per cent from 1895 to 1899, while the northwestern and western counties now containing 19 per cent of the total acreage of the State liave increased 51 per cent, a much greater rate of increase than that of the State for either the two previous decades, and undoubtedly coincident with the settling of new land as in the past. The average increase for the State is best shown by the thirty-seven northeastern and eastern comties, which produce 35 per cent of the crop of the State, which have been practically unaffected by the boll weevil up to 1904 , and which show an increase of 10 per cent over 1899 in 1904 .

In making these estimates the writer has been impressed with the fact that by no means as careful studies and statistics concerning the cotton acreage have been made as for the cotton product. The final reports upon the cotton crop as secured from the ginners by the census approximate the total yield as nearly as possible to do so. But the acreage is determined but once in ten years, and all acreages published for other years are necessarily but "estimates." Inasmuch as all estimates of the size of the crop of a given year, and the consequent market prices, must be based upon the acreage, it would seem that if some means could be devised whereby the acreage could be determined more accurately it would be of the greatest advantage to all interested in cotton. This was formerly done by means of reports upon acreage from each county made to the Texas Commissioner of Agriculture based upon an actual canvass of the acreage by the county assessors, as published for the years 1887 to 1895 . Only by some such well-organized system will accurate reports of the cotton acreage be secmred. That such reports of the acreage of this and other crops would be of the utmost value will hardly be questioned. Thousands of dollars are annually spent by brokers of staple crops to secure exactly this information. At the present time (July, 1905) there is considerable comment by brokers in the daily press that crop reports by the 
State or National govermments are mmecessary, as their information is not relied upon and brokers maintain their own organizations at much expense to secure crop estimates. But if crop reports are secured only by private parties, the market will be influenced by them and the farmer will be at the mercy of the brokers. Furthermore, it has been demonstrated that in most years the government crop reports mere nearly apjroximate the truth than any estimates secured by private parties. Correct crop reports and estimates by the governirient, State or National, enable the farmer to sell his crops to as good advantage as the broker may buy. Hence what is needed in the farmer's interest are better crop reports and better means for securing information, so that on all speculative crops the estimates may approach the actual conditions with the greatest possible accuracy. If the present discussion of the acreage of 'Texas, open to much error as the writer is well aware, owing to the sources from which the estimates were made, serves to bring out the necessity for more careful statistics for every county in Texas, his work will have accomplished much of its object.

\section{LOSSES DUE TO THE BOLL WEEVII.}

In order to estimate the amount of loss due to the boll weevil from year to year, as well as for a proper discussion of the acreage, it is necessary to group the counties, basing these groups upon the spread of infestation by the weevil. These groups are determined by (1) the year in which the counties first became intested, (2) the year in which counties first became injured, and (3) arbitrary geographical groups of uninfester or but slightly infested counties in which no appreciable injury has yet occurred. The spread of the weevil over the State up to August, 1901, as given by the United States Department of Agriculture and records of the State Entomologist, is shown in figure 1, and the groups of comnties based upon this in figure 2 . Of the southern counties infested by 1895 two groups are considered. The twelve of Southern Group \& were somewhat injured in 1899, but since then have yielded practically a normal crop each season until 1904, while those of Southern Group B, seven counties, have shown an increasing loss. Central Groups A and B were considered together mntil $190 \pm$ when Group A showed no decrease from the normal crop. Groups A and B, Central, include those comnties infested up to 1898 and first injured in 1900 . Central Group C comprises nineteen counties infested between 1898 and 1901, and first showing a decrease in 1902 and 1903 for the most part. Central Gromp D comprises four counties in north central Texas and three in soutlicastern, first infested in 190: and showing a decrease in 1903. Central Group E includes ten counties in west central Texas first infested in 190: but which have shown no appreciable loss from the previous normal crop. The remaining groups, Eastern, Northeastern, Western, Northwostern and Southwestern, though somewhat infested in part, showed no decrease up to 1903 , for which year these tables were first prepared. In 1904, six of the thirteen Eastern Gromp showed a decrease undoubtedly due to the weevil, which is shown in Table 1. It will be noted that except the different Central Groups, these groups of counties form fairly well-marked natural geographical divisions of 
the State. The Central Groups are made merely for convenience in estimating the damage from year to year and show very strikingly the fact often observed that it requires about two years after a county becomes infested with the weevil before it is serionsly injured.

In determining in what year a county first showed a decided decrease probably due to the weevil we have used the average yield per acre for that county from 1887 to 1895 as the average crop. If the average for the second year (or when the exact date of first infestation was unknown, any year beginning with the second year) was materially less than the average for that county, providing that the uninfested counties showed no decrease from normal yield in that year, that county was considered as first injured by the weevil in that year. If the uninfested counties showed a loss in a year, the difference in yield per acre was deducted from the apparent decrease of a county supposedly injured for the first time. Also where storms or floods were known to have seriously decreased the crop in certain counties, the injury was not attributed to the weevil in all cases. These latter factors and losses by other insects, it is of course impossible to accurately estimate. It is entirely safe to assume, however, that after the second year of infestation, the great bulk of the decrease is due to the weevil.

After determining the year in which each infested county first showed marked decrease as nearly as possible, and grouping them accordingly, it is an easy matter to as:emble the totals of the injured groups for each year as given in Table 1. The injured area for each year is shown in figures $3,4,5,6$ and 7 , together with lines showing the progress of previous infestation as related to this injury.

The total loss due to the weevil each year was determined as follows: The yield per acre for the injured and uninjured areas was determined. The yield per acre for the injured counties was then subtracted from their normal yield per acre from 1887 to 1895 , giving the "apparent" decrease for the injured area. The vield per acre for the uninjured area for 1899 was then compared with its arerage yield per acre from 1887 to 1895 , and the gain or loss computed in bales and bales per acre. Subsequent to 1899 , instead of the yield per acre for the uninjured area from 1887 to 1895 , was used the average of the years 1887 to 1895 and the years from 189! to date inchusive, omitting 1896-189S in which years there are no means of forming estimates by counties. The large, crop of 1900 increased this arerage yield per acre for the uninjured area nuaterially, making it 0.02 bales more per acre and in 1904 it was 0.05 bales per acre greater than the average from $188 \%$ to 1895 . If there was a gain in bales per acre in the uninjured area, the area of the injured counties was multiplied by the number of bales per acre gain for the uninjured counties and the result added to the "apparent" decrease for the injured counties, giving the "total bales decrease in the injured counties." If, on the other hand, the uninjured counties showed a loss from the average of the previous years, the loss in bales per acre was multiplied by the acreage injured and the result deducted from the "apparent" injury. Thus due weight is given to the seasonal increase or decrease of the uninjured area in reckoning the amount of injury. Had the "apparent" decrease been increased by the difference between the yield per acre of the uninjured area for a given year and its average 
between 1887 and 1895 the "total decrease" would have been very much greater than by using the average of those years plus that of years since 1899 as given in column 9, Table 1. Had the average from 1887 to 1895 alone been thus used there would have been shown a total decrease of 325.000 bales against 258,489 given in $1902,725,000$ against 500,000 given in 1903, and about the same difference in 1904 .

From the "total decrease" must be subtracted the losses due to storms, floods, and insects such as the boll worm, which is practically the only insect causing any large loss in the weevil injured area. 'These latter factors are exceedingly difficult to estimate. In 1899 there was a serious flood of the Brazos river, but it did not affect the injured counties. The Department of Agriculture estimates the injury due to the Galveston storm at 68,000 bales, and we have allowed $\$ 6,810$ bales loss due to it and boll worm in 1900 .

It should be noted also that had we computed the loss by using the acreage figures of the Department of Agriculture, which are uniformily larger than our estimate, the loss due to the weevil would have been much larger, as the yield per acre of the injured counties would have been rery much less than eren the apparent differences in total acreages would indicate, for the Department of Agriculture has estimated many of the injured counties as haring actually increased their acreage dus'ing fire years in which, in Table 2 , we have indicated a decrease, which is supported by the best information obtainable.

The methods employed in this estimate of the losses due to the reevil have thus been purposely made as conservative as possible. while at the same time making due allowance for the increased or decreased production for the uninjured part of the State in determining the difference between the actual crop and what the normal crop should be for the injured area for the same years.

Let ns consider the recrease due to the weevil year by year somewhat more in detail.

In 1899, the eighteen southern counties, practically those lying south of the Southern Pacific Railroad, having j per cent of the acreage and producing $t$ per cent of the crop of that year, yielded but 0.28 bales per acre against a normal 0.43 bales per acre, while the balance of the State showed a decrease of but 0.15 bales per acre. The total decrease of these counties was, therefore, 0.135 bales per acre, or $174,71 \mathrm{~S}$ bales, at least 150.000 bales of which may be due to the weevil. It may be noticed that these counties were all infested $\mathrm{mp}$ to 1895 . During 1896 and $189 \%$ the weevil did not spread beyond the area infested in 1895 . Probably nearly as large a loss occurred in these counties in 1898, were the acreage or crop figures available by counties for that year.

In 1898 the weevil adranced as for north as Burleson and Brazos counties (fig. 1), and as a result two years later, in 1900, a marked decrease is found in the production of all these counties, as graphically shown in figure 4. In 1900, however, the counties of Southern Group A produced better than a normal crop and Southern Group B practically a normal crop, though 0.15 bales per acre less than the increase in the balance of the State warranted. The thirteen counties of Central Group $\mathrm{AB}$ injured for the first time, yielded but 0.26 bales per acre, just half per acre that produced in the balance of the State. Together the 
twenty injured counties showed a decrease of 0.30 bales per acre or 311,810 bales. From this must be deducted the loss due to the Galveston storm throughout south central Texas, which reduces the total loss to probably 200,000 bales. In 1900 the injured area was 15 per cent of the acreage of the State, three times that of 1899, and the injured area produced 18 per cent of the crop of 1899 .

In 1901 the injured territory remained practically the same as in 1900, Caldwell county being the only one added. Though no records exist of the spread of the weevil in 1899, it is evident from the increased injury in 1902 and the area infested in 1901 that there was a considerable advance of infestation in 1900 and it seems probable that a very small migration in 1899, as in 1896-9\%, accounts for there being no larger area injured in 1901. The injured counties showed the same "apparent" decrease in 1901 as in 1900, but owing to a decrease of 0.05 bales per acre for the balance of the State, the total real decrease was but about one-third that in 1900 , or 100,920 bales. In other words, the injured counties compared with the yields of the other counties in the State made a gain of 0.20 baks per acre in 1901 over 1900 , though they actually produced but 0.01 bales per acre more.

In $190^{\circ}$ about luif of the counties infested between 1898 and 1901 were added to the injured area of 1901 , comprising nine of the counties of Central Group C, making 26 per cent of the total acreage injurexl, which acreage produced 3i per cent of the total crop of 1899 . It was, therefore, in 19(1)' that the most productive counties in Texas were first injured, as the prer cent of the total product produced in the injurer comnties was about :3.) per cent greater than the per cent of the total acreage occupied by them.

The decrease in production in the injured counties was also more serious than previonsly, the thirty-two injured counties producing but $0.2 S$ bales per acre, though owing to a decrease from normal of 0.03 bales per acre for the balance of the State, the actual decrease was 0.14 bales per acre, the same as in 1901, or 258,489 bales. From this must be deducted loss from the boll worm amounting to 5 per cent according to Prof. Mally's estimate (see his Report on the Boll Worm, 1902), which would be approximately 25,500 bales, and loss due to the Brazos river flood of at least an equal amount, so that the probable total loss attributable to the weevil was aluout 200,000 bales, about the same as in 1900 .

The year 1903 witnessed an exen greater relative enlargement of the injured area than 1902. All of the territory infested in 1901 and much of that which had been first reported infested in 1902, showed a marked decrease which was more striking in view of the fact that the balance of the State showed slightly better than a normal crop. Indeed, the advance of infestation and injury by the weevil may well be compared to the waves radiating from a pebble cast in water, the second following the first in concentric and ever-increasing circles, which lines of advance have, however, pushed more rapidly to the north in the case of the weevil; 38 per cent of the 1903 acreage, which produced 50 per cent of the crop in 1899, but only 26 per cent in 1903, and including soine of the largest and most productive cotton counties of Texas, was injured in 1903. The injury in the combties previously injured, as 
well as in the counties injured for the first time, was more serious than ever before, there being a yield of but 0.23 bales per acre in the 49 injured counties against 0.43 previously. This was no doubt largely due to the exceedingly late and wet season throughout the most of the State, favorable to the development of the weevil. The decrease for the injured counties amounted to 0.21 bales per acre or a total of 580.612 . From this should be subtracter injury by the boll worm which was unusually serere, especially in Central Group D, probably amounting to 12 per cent for the whole forty-nine counties, learing in round numbers 500,000 bales which may safcly be attributed to injury by the boll weevil. The Southern Group A comnties continued to show a normal crop in spite of the weevil.

The injury in 1904 was quite different in many respects from any previous years. 'The total acreage injured slowed a decrease from that of 1903, due to the fact that nine counties in central Texas, Central Group A, Bell. Hass, Limestone and Williamson, having a total acreage of 8 per cent that of the State, and producing nearly 10 per cent of the total crop, proslueed better than a normal crop compared with the uninjured counties, though in 1903 they had all shown serious injury. In contrast to this enconlaging feature, the twelve counties of Southern Groul, i, owing to a rery mild winter, early spring, and allowing stubble cotton to remain over winter upon which the weevils commeneed to multiply early, produced but half a normal crop, for the first time showing serious injury since 1899. In addition to the injured area of 1903 were seven counties in east Texas and ten southwestern counties, the outer limits of the area injured in $190 t$ being almost exactly those infested in 1902 , except for the ten counties of Central Group E. which showed a better than normal crop, though many of them had been well infested in 1903 and all more or less in 1902. Together the sixty-nine injured counties showed an "apparent" decrease of 0.14 bales per acre, which with the increase of 0.08 bales per acre for the balance of the State gave 0.22 bales per acre total loss, practically the same as in 1903 , making $586, \pm \% 8$ bales decrease, slightly larger than for 1903. In $190 t$ there was comparatively little injury from the boll worm and loss from flood, so that 550,000 bales may safely be charged to the boll weeril. Had the nine counties which preriously had shown a loss, but in $190+$ made better than a normal crop, showed as much injury as those surrounding them, the total loss would have approximated ro0,000 bales. Had the ten counties of Central Group 3 been injured as much as the seven eastern counties after two rears of infestation, there would have been 135,000 bales further decrease. In other words, had the wecril been as injurious in the territory infested two years or over as previously, there would have been a Joss of $8 \% 5,000$ bales of cotton due to the weevil in 1904. That this was not the case and that a phenomenal crop was made was undoubtedly due to the extremely favorable season, an early spring, hot summer, and a late growing scason. These favorable weather conditions made possible the best possible results from the "cultural methods" of preventing injury by the weevil, consisting of exrly planting, early varieties, and thorough, continned cultivation. These methods were widely practiced by progressive planters and undoubtedly were a large factor in 
producing a crop above the average for the uninjured counties, including nine of the largest counties which had previonsly shown serious loss, as well as lessening the loss in other counties. It should be borne in mind, however, that in spite of the large total crop the loss to over one-third the acreage of the State was as severe as in 1903 and was fully one-half the crop of those counties. Had there been no loss by the boll weevil in 1904 Texas would probably have produced $3,750,000$ bales of cotton.

Footing up the bill which the boll weevil has enforced payment by the State of Texas we find a total of $1, \hat{r} 25,000$ bales loss during the past six years. Since its arrival in Texas $2.000,000$ bales, worth nearly $\$ 100,000,000$, have undoubtedly been lost due to its devastations. If we consider the average yield of the injured territory as .438 bales per acre (averaging the six years). there has been a decrease of 43.2 per cent of the crop in this territory, arcraging 0.18 a bales per acre a year. In other words, it now takes $1 . i 1$ acres to produce the crop of one acre before the arrival of the weevil, or where 2.28 acres were formerly required to produce a bale of cotton 3.9 acres are now necessary.

\section{THE FUTTRE OLTLOOK.}

What do these statistics teach which will tend to mitigate a present loss of from $\$ 20,000,000$ to $\$ 25,000.000$ ?

First, that as a whole this loss is increasing and that with a season such as 1903 the area now well infested by the weevil might show a loss of from 900,000 to $1,000,000$ bales.

Second, that in extreme southern Texas the weevil rarely seriously injures the crop if stubble cotton is not allowed to remain over winter in mild winters.

Third, that there seems a probability that the weevil may not be so injurious in the restern cotton comnties.

Fourth, that in a farorable or arerage season a fair crop can be grown in spite of the weevil by the use of better methods, such as early planting, fertilization, better-more productive and earlier-varieties, more thorough, frequent and continued cultivation. It remains for future crops to show how much value these methods will have in an unfarorable year. It is the belief of the writer that these methods are of the greatest value and should be practiced intelligently by everyone, but he is prone to feel that not until the increase of the weevils is actually checked by the general destruction of the cotton stallis before frost in the fall, by the people generally, will there be a material reduction of loss by the weevil in at least one year out of three and possibly half of the time. It is high time that the Strte regulated the matter of the destruction of stumpage cotton in south Texas, and it should at least make experiments on a sufficiently large scale to demonstrate beyond any manner of doubt and to convince the most skeptical that the fall destruction of the stalks throughout the State is or is not the best means of controlling the pest, as advocated by all who have given it careful study.*

*Suggestions to this end, with a full discussion of the subject, were published by the writer in Farm and Ranch, October 8, 1904. 


\section{LEGENDS FOR FIGURES ACCOMPANYING ARTICLE ON IN- JURY TO COTTON CROP BY BOLL WEEVIL.-SANDERSON.}

Fig. 1. Map of 'Texas showing the gradual spread of the boll weevil to August, 1904. -...- lines indicate probable infestation, but not actually reported. Lines for 1894, 1895, 1898, 1901 are those published or recorded by the Bureau of Entomology, U. S. Department of Agriculture, as are parts of those for 1903 and 1904. The line for 1902 is from records made mostly by Prof. F. W. Mally. The black line at the west indicates the western boundary of cotton culture in 1899 .

Fig. 2. Map of Texas showing the groups of counties used in tabulation. The counties underlined are part of those included under "Far Western," Table IV.

Fig. 3. Counties injured by the boll weevil in 1899 are shaded.

Fig. 4. Counties showing a marked decrease in yield due to the boll weevil in 1900 and 1901 are shaded.

Fig. 5. Counties showing a marked decrease in yield due to the boll weevil are shaded.

Fig. 6. Counties showing a marked decrease in yield due to the boll weevil in 1903 are shaded.

Fig. \%. Counties showing a marked decrease in yield due to the boll weevil in 1904 are shaded. Counties injured by the weevil in 1903 which yielded better than a normal crop in 1904 are underlined. 



LIBRARY OF CONGRESS

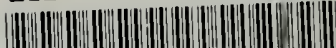

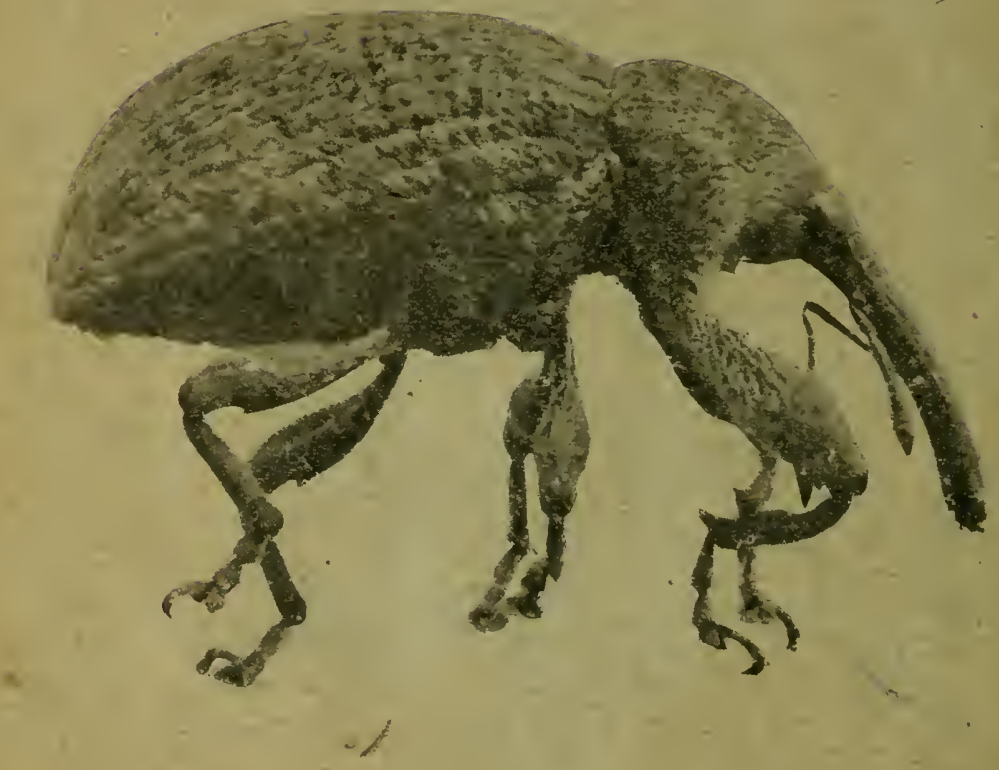

